\title{
Turbulent inflow conditions for large-eddy simulation based on low-order empirical model
}

\author{
Laurent Perret, ${ }^{1, a)}$ Joël Delville, ${ }^{2}$ Rémi Manceau, ${ }^{2}$ and Jean-Paul Bonnet ${ }^{2}$ \\ ${ }^{1}$ Laboratoire de Mécanique des Fluides (LMF), UMR CNRS 6598, Ecole Centrale de Nantes, \\ 1 rue de la Nö̈ BP 92101, F-44321 Nantes Cedex 3, France \\ ${ }^{2}$ Laboratoire d'Etudes Aérodynamiques (LEA), ENSMA, CNRS, CEAT, Université de Poitiers, \\ 43, route de l'aérodrome, F-86036 Poitiers, France
}

(Received 30 October 2007; accepted 3 June 2008; published online 22 July 2008)

\begin{abstract}
Generation of turbulent inflow boundary conditions is performed by interfacing an experimental database acquired by particle image velocimetry to a computational code. The proposed method ensures that the velocity fields introduced as inlet conditions in the computational code present correct one- and two-point spatial statistics and a realistic temporal dynamics. This approach is based on the use of the proper orthogonal decomposition (POD) to interpolate and extrapolate the experimental data onto the numerical mesh and to model both the temporal dynamics and the spatial organization of the flow in the inlet section. Realistic representation of the flow is achieved by extracting and modeling independently its coherent and incoherent parts. A low-order dynamical model is derived from the experimental database in order to provide the temporal evolution of the most energetic structures. The incoherent motion is modeled by employing time series of Gaussian random numbers to mimic the temporal evolution of higher order POD modes. Validation of the proposed method is provided by performing a large-eddy simulation of a turbulent plane mixing layer, which is compared to experimental results. (C) 2008 American Institute of Physics.
\end{abstract}

[DOI: $10.1063 / 1.2957019$ ]

\section{INTRODUCTION}

Due to significant developments in computational methods over the past few decades, numerical simulations are now widely employed to investigate a large variety of flows. These improvements enable consideration of more and more complex flows that imply complex physical phenomena and geometries. However, in spite of these advancements, a number of flows are still too expensive to simulate because of their strong unsteady character that must be taken into account to reproduce the main flow features. To overcome these issues and limit the computational cost, new strategies that aim at simulating only the region of interest of the flow have recently been developed. These approaches are, for instance, applied in the framework of zonal hybrid Reynolds averaged Navier-Stokes (RANS)/large-eddy simulation (LES) calculations (see Sagaut et al. ${ }^{1}$ for a comprehensive recent review of these approaches) where the use of LES is limited to the region in which the flow characteristics can only be obtained by an unsteady simulation. A critical issue that arises in such strategies is the imperative need for correct specification of all the boundary conditions of the spatial domain for the unsteady computation. Except if the computational domain includes the whole transition of the flow from laminar to turbulent state, one must be able to prescribe inlet conditions that meet all the LES requirements. Consequently, generating proper inlet conditions for unsteady simulations such as LES of spatially developing flows requires the generation of bidimensional vector fields evolving

\footnotetext{
${ }^{a)}$ Electronic mail: laurent.perret@ec-nantes.fr.
}

in time in agreement with the spatiotemporal dynamics of the flow. This implies that the modeled vector fields present the correct one- and two-point statistics in space as well as in time, in agreement with the targeted flow, with the correct spatial and temporal coherence to reproduce realistic turbulent structures. If the inlet conditions do not fulfill all these requirements, the simulated flow will undergo an adaptation in a region of the computational domain to regenerate correct turbulent eddies.

In the framework of simulating incompressible spatially developing turbulent flows, several methods with different degrees of sophistication and computational costs have been proposed to address the issue of generating correct inflow conditions. The simplest method, derived from the technique used for simulations in which the transition to turbulence is computed, consists in generating synthetic signals with imposed energy level and energy spectra via the use of sequence of random numbers. ${ }^{2,3}$ Because of its relative simplicity, this method presents a major limitation: the generated turbulent fluctuations do not contain any phase information representative of the real eddies. Consequently, the use of this method always leads to the existence of a non-negligible adjustment region in which the computational code regenerates realistic turbulent structures. It should be emphasized that this adjustment region corresponds to a process of the perturbation that has no physical meaning.

In order to simulate a turbulent flow over a bump, Sandham et ll $^{4}$ designed a new method to generate synthetic turbulence based on the introduction of specific disturbances, the nature of which depending on the wall-normal location. These disturbances representing low-speed streaks or large 
coherent structures (that are, respectively, found in the inner and outer layer of wall-bounded flow) contain the correct phase information necessary to represent realistic turbulent structures. Random noise is superimposed to break the remaining symmetries. An analogous approach is used by Jarrin et al. ${ }^{5}$ to generate synthetic eddies in the inlet section. By enabling the specification of the spatial and temporal characteristics of each eddy, their method is able to reproduce first and second order one-point statistics and autocorrelation functions.

In order to obtain inflow data that present the real spatiotemporal dynamics of a turbulent flow, methods based on the modification of periodic conditions used in simulations of temporally developing flows have been developed. These methods, such as those developed by Lund et al. ${ }^{6}$ and Ferrante and Elghobashi ${ }^{7}$ for wall-bounded flows, consist in extracting velocity data from a downstream section of the simulation and reintroducing them at the inlet section after a rescaling operation that ensures that inlet conditions present the correct fluctuation levels and spatial scales. These methods are inherently limited to flows in which part of the computational domain can be devoted to the recycling procedure, where the rescaling operation is possible. A more efficient method in terms of quality of the generated inflow conditions is based on the use of a separate precursor calculation, presenting a configuration simpler than the flow to be eventually simulated. Velocity data are extracted from this simulation and directly used as inlet conditions for the main computation $^{8}$ or after some rescaling adjustments as performed by Schlüter et al. ${ }^{9}$ For a detailed review and comparisons of the above-presented methods for generating inflow conditions, see Keating et al. ${ }^{10}$ and Sagaut et al. ${ }^{1}$

Other recent approaches rely on the fact that coherent structures embedded within the flow play a dominant role in the spatiotemporal dynamics of turbulent flows. Indeed, it is now well established that they have a strong influence on mixing processes, development, and stability of the flow. Based on this fact, Johansson and Andersson ${ }^{11}$ modified the precursor calculation method by using a very-large-eddy simulation (VLES) to produce inflow conditions for both a direct numerical simulation (DNS) and a LES of a channel flow. The VLES was designed to simulate the dynamics of the most energetic coherent structures of the flow extracted by proper orthogonal decomposition (POD) from either a DNS or a LES. Random small-scale motion was added in order to represent some energy in the high wave-number part of the spectrum. These random small-scale modes consisted of randomly varied POD modes not included in the VLES. Their study demonstrated the potential of such an approach by drastically reducing the computing time required by the VLES compared to a full DNS or a well-resolved LES. However, the necessity of obtaining the POD basis derived from the full two-point correlation tensor is still an important drawback.

Another strategy, which also relies on the fact that the dynamics of the coherent structures must be properly taken into account, has been proposed by Druault et al., ${ }^{12}$ which aims at generating realistic inlet conditions from an experimental database. In their work, Druault et al. ${ }^{12}$ take advan- tage of simultaneous multipoint measurements performed with hot wires to obtain time-varying information on the coherent structures of a turbulent mixing layer. An optimal spatial interpolation/extrapolation procedure based on the POD and the linear stochastic estimation (LSE) enables the estimation of the three-component velocity field representing the most energetic turbulent structures present in a section that corresponds to the inlet section of the LES. This study demonstrated the importance of taking into account the twopoint spatial and temporal correlations of the targeted flow when generating inflow data for LES. However, some limitations were pointed out by the authors. From an experimental point of view, it can be difficult to build and implement a rake of several dozens of $X$ hot wires. This kind of arrangement can lead to some blocking effect in the wind tunnel cross-section. Moreover, the spatial sampling possible with this technique is intrinsically limited and far from that required by the simulation. It also turns out that the combination of the POD and the LSE acts as a filter both in energy and frequency, leading to a lack of energy and a deterioration of the level of the Reynolds shear stress $\left\langle u^{\prime} v^{\prime}\right\rangle$.

More recently, Perret et al. ${ }^{13}$ extended these concepts to the use of a database acquired by stereoscopic particle image velocimetry (SPIV). Contrary to the previous method based on hot-wire measurements, the SPIV database presents a spatial resolution in agreement with the computational mesh but requires an ad hoc temporal modeling procedure to overcome the fact that SPIV measurements cannot provide any time evolution of the velocity data for the flow under study. In this study, interfacing the SPIV data to the computational code was performed by using a POD approach to perform both the extrapolation and interpolation of the experimental data onto the computational grid. The temporal dynamics of the velocity field was modeled via time series of random numbers with prescribed spectra. This method was employed to perform a LES of a turbulent mixing layer that demonstrated its feasibility. However, even if promising, this first attempt was affected by the lack of phase information of the temporal modes due to the use of random time series. This absence of realistic turbulent structures at the inlet of the computation led to a rapid drop of the energy level downstream of the inlet section and the need for the simulation to regenerate turbulent structures. Consequently, the obtained numerical results revealed that an adjustment region is still present at the beginning of the computational domain. It should be noted here that the use of the time-resolved PIV (TR-SPIV) could be a solution to overcome the absence of time history in classical PIV data. However, the still limited acquisition frequency of TR-SPIV setup can prevent the study of high Reynolds number flows.

The purpose of the present paper is to present an extension and improvement of the work initiated by Perret et al. ${ }^{13}$ by employing a low-order dynamical system ${ }^{14}$ (LODS) identified from a dual-time SPIV (DT-SPIV) database in order to correctly model the spatiotemporal dynamics of the most energetic turbulent structures. The underlying idea is to use a LODS in order to provide the correct phase information to the first POD modes representing the most energetic coherent structures in a section corresponding to the inlet of the com- 
putational domain. The temporal dynamics of the remaining modes that can be viewed as representative of the incoherent part of the velocity field is modeled by synthetic random time series with realistic energy spectra. The main goal of the present study is to provide a method for the generation of inlet conditions that is accurate in terms of the characteristics of the reproduced flow (e.g., energy levels, two-point correlations, spectral content, phase between modes, etc.) and that does not take advantage of any particular feature of the flow under study. Thus, unsteady three-dimensional (3D) turbulent inlet conditions for LES are entirely derived from an experimental database that only contains uncorrelated samples. The development of the dynamical model used to reproduce the large scale dynamics requires only uncorrelated samples of both velocity and acceleration fields. ${ }^{14}$ Such quantities can be obtained from measurements performed by DT-SPIV,${ }^{15}$ e.g., containing no time history. Consequently, if the acquisition of the database is feasible from an experimental point of view, the method presented here can be employed when dealing with flows of complex configuration that cannot be handled by using a recycling method or running a precursor simulation.

The feasibility of the proposed method is demonstrated in the present paper by performing a LES of a turbulent mixing layer, the parameters (e.g., Reynolds number and velocity ratio) of which are exactly the same as those of the flow investigated experimentally to build the inlet condition database. In Sec. II, the flow configuration and the experimental setup used to acquire the required database are presented. Section III gives a detailed presentation of the methods developed to perform the temporal modeling and the spatial adjustment of the generated inflow velocity fields as well as their characteristics. The main features of the generated inflow data for the present mixing layer LES are investigated in Sec. IV. Eventually, a detailed analysis of the LES results performed with the present inlet conditions is proposed in Sec. V.

\section{FLOW CONFIGURATION AND EXPERIMENTAL SETUP}

\section{A. Characteristics of the mixing layer}

The experiment designed to acquire the database needed for the generation of inflow data is performed on a turbulent plane mixing layer. This flow is the result of mixing two air streams of different velocities downstream of a splitter plate with a $12^{\circ}$ bevelled trailing edge, generated in a $0.3 \times 0.3 \mathrm{~m}^{2}$ closed-loop wind tunnel. The longitudinal axis $x$ is parallel to the mean flow direction, $y$ is the axis normal to the splitter plate, and the tranverse axis is $z$. The velocity components $u$, $v$, and $w$ are, respectively, associated with the axes $x, y$ and $z$. The origin of the coordinate system is located at the center of the splitter plate trailing edge. The high and low speed velocities of this two-dimensional subsonic mixing layer are, respectively, $U_{a}=35.2 \mathrm{~m} / \mathrm{s}$ and $U_{b}=23.8 \mathrm{~m} / \mathrm{s}$, corresponding to a velocity ratio $r=0.67$. The boundary layers developing on each side of the splitter plate were turbulent. The section retained to perform measurements and which will correspond to the inlet section of the computational domain

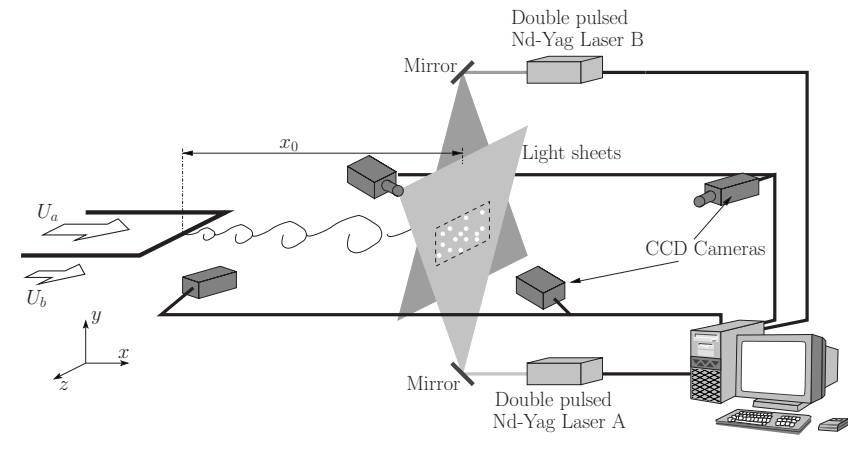

FIG. 1. DT-SPIV setup.

in the following is located at $x_{0}=300 \mathrm{~mm}$ downstream of the splitter plate trailing edge. This location corresponds to the beginning of the self-similarity region of the mean flow. At this location, the Reynolds number based on the local vorticity thickness $\delta_{\omega 0}=18.7 \mathrm{~mm}$ and the mean velocity $U_{m}$ $=\left(U_{a}+U_{b}\right) / 2$ is $\operatorname{Re}=\delta_{\omega 0} U_{m} / \nu \simeq 36000$. For a more detailed description of the flow and its characteristics, the reader is referred to the work of Perret et al. ${ }^{15}$ and Perret. ${ }^{16}$

\section{B. Measurement apparatus and procedure}

In the present study, the dynamics of the coherent structures in the measurement plane is modeled by LODS. This model is directly derived from the experimental database by using the identification method proposed by Perret et al. ${ }^{14}$ which is described in Sec. III. This method requires access to instantaneous velocity fields as well as their temporal derivatives. For that purpose, an experimental setup composed of two synchronized SPIV systems was designed to perform DT-SPIV measurements. This setup aims at providing pairs of bidimensional three-component velocity fields at the same location but shifted in time. By carefully choosing the time delay $\tau$ between the two successive velocity fields, this method enables us to compute time derivatives with a finite difference scheme. Only a brief description of this experimental setup (presented in Fig. 1) is given in this section. A full description, an evaluation of the measurement uncertainties, as well as a methodology based on the analysis of the spectral error distribution to select the proper time delay to compute accurate time derivatives are provided in Perret et al. ${ }^{15}$

The measurements are performed in a section normal to the mean flow, in the $y-z$ plane, with two synchronized SPIV systems labeled $A$ and $B$ that provide velocity fields $\mathbf{u}^{A}(y, z, t)$ and $\mathbf{u}^{B}(y, z, t+\tau)$, respectively. To illuminate the seeded flow in this section, two independent double-pulsed Nd:YAG lasers are employed. Optical separation between the two independent systems is achieved via the use of a different linear polarization of the light generated by each laser. Two $1350 \times 1049$ charge coupled device cameras are associated with each laser system. They are mounted in a stereoscopic angular configuration with Scheimpflug correction and are fitted with polarizing filters so that each camera could only be illuminated by the light diffused by the laser it is associated with. Olive-oil droplets generated from a 
Laskin nozzle are employed as seeding material. The generated particle diameter presents a distribution with a mean diameter close to $1 \mu \mathrm{m}$. The temporal synchronization of all these components as well as PIV processing of the particle images are performed with the LaVision acquisition software. The reconstruction of the three-component vector fields from pairs of images of each stereoscopic system is performed by using the $3 \mathrm{D}$-calibration method proposed by Soloff et al. ${ }^{17}$

A cross-correlation algorithm based on fast Fourier transform (FFT) is used with an adaptative multipass interrogation window technique. The final size of the interrogation window is $32 \times 32$ pixels $^{2}$ with $50 \%$ overlapping of data, leading to a spatial resolution of $0.088 \delta_{\omega 0}$ and a SPIV measurement domain of $4 \delta_{\omega 0} \times 6.1 \delta_{\omega 0}$ in the $y$ and $z$ directions. Besides, in the mixing layer, the average spanwise spacing between vortices of longitudinal axis is of the order of $2 \delta_{\omega 0}$ (e.g., $\frac{2}{3}$ of the average longitudinal spacing between two successive Kelvin-Helmoltz structures).$^{18}$ Thus, the size of the SPIV domain is large enough to capture most of the large scales of the flow in both the vertical and spanwise directions while maintaining a spatial resolution sufficient to correctly sample the most energetic structures. Given the flow characteristics, the time delay retained for the PIV processing between two pulses of the same laser system is set to $20 \mu$ s. The time delay between the two acquired velocity fields used to compute time derivatives is chosen on the basis of the analysis of prior hot-wire velocity measurements and set to $80 \mu$ s to minimize the influence of the measurement noise. ${ }^{15} N_{s}=2000$ pairs of stereoscopic velocity fields are acquired at a rate of $1 \mathrm{~Hz}$. It must be noted here that in the present study, the characteristic frequency of the KelvinHelmoltz structures is of the order of $400 \mathrm{~Hz}$ (based on the Strouhal number $\left.S t=f \delta_{\omega 0} / U_{m}=0.33\right),{ }^{19}$ showing that the acquired velocity pairs are totally uncorrelated in time with each other.

\section{INFLOW DATA GENERATION}

In this section, the proposed method for the generation of unsteady turbulent inflow conditions for LES is described. Two issues are involved in such an approach. First, one must be able to properly model the temporal dynamics of the flow in the inlet section of the computational code. Second, the experimental data have to be extrapolated and interpolated onto the grid of the computational domain. It implies not only that the generated velocity fields present the correct spatial resolution but also that they must be consistent with all the boundary conditions retained for the simulation. These two main steps rely on the use of the POD, which enables the decomposition of the velocity field into two independent sets of spatial and temporal modes. Thus, the temporal modeling of the flow and the spatial adaptation of the experimental data to the numerical grid can be performed independently. In the following section, the POD is first briefly described. The developed strategies to both reproduce the temporal dynamics of the flow in the measurement section and interpolate and extrapolate the experimental data to the computational grid are described next.

\section{A. POD}

The POD was proposed by Lumley ${ }^{20}$ as an objective method to study the coherent structures of turbulent flows. Only a brief description is presented here; more details can be found in the work of Berkooz et al. ${ }^{21}$ and Delville et al. ${ }^{19}$

Lumley proposed to identify the coherent structures embedded into turbulent flows as the structure $\phi(\mathbf{X})$ with the largest mean-square projection onto the velocity field $\mathbf{u}(\mathbf{X}, t)$, e.g., with optimal energy content. This maximization process leads to the integral eigenvalue problem

$$
\int_{\mathcal{D}} R_{i j}\left(\mathbf{X}, \mathbf{X}^{\prime}\right) \phi_{j}^{(n)}\left(\mathbf{X}^{\prime}\right) d \mathbf{X}^{\prime}=\lambda^{(n)} \phi_{i}^{(n)}(\mathbf{X}),
$$

where the kernel is the two-point velocity correlation tensor, $R_{i j}\left(\mathbf{X}, \mathbf{X}^{\prime}\right)=\left\langle\mathbf{u}_{i}(\mathbf{X}, t) \mathbf{u}_{j}\left(\mathbf{X}^{\prime}, t\right)\right\rangle,\langle\cdot\rangle$ being the ensemble average operator, equivalent to the temporal average in the present case, and the eigenvalue $\lambda^{(n)}$ represents the turbulent energy contained in the $n$th mode. The velocity field can then be expressed as a linear combination of its POD eigenfunctions $\phi_{i}^{(n)}(\mathbf{X})$,

$$
\mathbf{u}_{i}(\mathbf{X}, t)=\sum_{n=1}^{\infty} a^{(n)}(t) \phi_{i}^{(n)}(\mathbf{X})
$$

The projection coefficients $a^{(n)}(t)$ are given by

$$
a^{(n)}(t)=\sum_{i=1}^{3} \int_{\mathcal{D}} \mathbf{u}_{i}(\mathbf{X}, t) \phi_{i}^{(n)}(\mathbf{X}) d \mathbf{X}
$$

and are uncorrelated

$$
\left\langle a^{(n)}(t) a^{(m)}(t)\right\rangle=\lambda^{(n)} \delta_{n}^{m} .
$$

In the present study, due to the fact that the measurements were performed by SPIV, the POD approach proposed by Sirovich, ${ }^{22}$ referred to as snapshot POD, was preferred to perform the POD decomposition. This version is indeed more adapted to data that are well resolved in space with a limited number of time samples. Besides, as the measurement domain is reduced to the $y-z$ plane, the spatial eigenvectors are three-component functions of two variables: $\phi_{i}^{(n)}(y, z), i=1,2,3$. As $N_{s}$ PIV samples were used, $N_{s}$ POD modes were obtained from the resolution of the eigenvalue problem (1).

\section{B. Temporal dynamics modeling}

Given the fact that the whole spatiotemporal dynamic of the flow cannot be modeled in a simple manner, the order of the problem is reduced by using the triple decomposition concept as introduced by Reynolds and Hussain. ${ }^{23}$ These authors proposed to separate the velocity field into three components, mutually uncorrelated: the mean flow $\langle\mathbf{u}\rangle$, the coherent part $\mathbf{u}_{c}$, and the random contribution $\mathbf{u}_{r}$. In the present study, the POD is used to separate the coherent part from the random part of the velocity field. Thus, the contribution of the $N_{t r}$ first POD modes to the velocity field represents the coherent part 


$$
\mathbf{u}_{c}=\sum_{n=1}^{N_{t r}} a^{(n)}(t) \phi^{(n)}
$$

and the contribution of the remaining $N_{s}-N_{t r}$ POD modes represents the random part

$$
\mathbf{u}_{r}=\sum_{n=N_{t r}+1}^{N_{s}} a^{(n)}(t) \phi^{(n)} .
$$

The velocity field can then be reconstructed as

$$
\mathbf{u}=\langle\mathbf{u}\rangle+\mathbf{u}_{c}+\mathbf{u}_{r}=\langle\mathbf{u}\rangle+\sum_{n=1}^{N_{t r}} a^{(n)}(t) \phi^{(n)}+\sum_{n=N_{t r}+1}^{N_{s}} a^{(n)}(t) \phi^{(n)}
$$

Different strategies to model the temporal coefficients $a^{(n)}(t)$ of both the coherent part and the random part can be employed, depending on which part of the flow is considered. As described below, the temporal POD coefficients representing the dynamics of the coherent part of the flow (e.g., the most energetic structures), denoted $\widetilde{a}^{(n)}(t)$, are modeled by a LODS identified from the DT-SPIV database. The temporal coefficients related to the random part, referred to as $b^{(n)}(t)$, are modeled by a series of random numbers. Finally, a modeled velocity field can be reconstructed as a combination of both the coherent and random parts such as

$\widetilde{\mathbf{u}}=\langle\mathbf{u}\rangle+\mathbf{u}_{c}+\mathbf{u}_{r}=\langle\mathbf{u}\rangle+\sum_{n=1}^{N_{t r}} \widetilde{a}^{(n)}(t) \phi^{(n)}+\sum_{n=N_{t r}+1}^{N_{s}} b^{(n)}(t) \phi^{(n)}$

In the present study, when deriving the LODS to model the temporal behavior of the first $N_{t r}$ POD modes, two difficulties arise: (1) the problem of the model stability inherent to the fact that only a limited number of modes are kept, then suppressing the influence and the interaction of the other modes, and (2) the fact that the model parameters are derived from an experimental database that is inevitably affected by the measurement uncertainty. As a matter of fact, in the present study, the parameters of the low-order model that drive the dynamics of the POD coefficients $\widetilde{a}^{(n)}(t)$ are directly derived from the experimental database via a leastsquares approach. This parameter estimation involves two sets of velocity fields measured by two distinct (but synchronized) SPIV systems that are both affected by random noise. This measurement noise affects not only the evaluation of the velocity fields but also the evaluation of the acceleration fields that are required to estimate the model parameters. As it will be shown later, the experimental uncertainty has an increasing influence when high order POD modes are taken into account and leads to more and more biased estimation of the LODS parameters. The number of POD modes retained in the LODS, $N_{t r}$, is thus limited to keep the influence of the measurement error as low as possible. However, the limited number of modes retained in the LODS developed does not prevent the simulation to produce satisfactory results.

\section{Low-order model identification from PIV database}

Principles. In the present study, temporal evolutions of the velocity fluctuations are modeled through the POD projection coefficients $a^{(n)}(t)$. A LODS is derived from the DT-SPIV database acquired with the experimental setup presented in Sec. II in order to model the temporal behavior of the $N_{t r}$ first POD modes representing the coherent structures of the flow [Eq. (8)]. The method used for deriving the LODS is briefly presented here, readers being referred to Perret et al. ${ }^{14}$ for more details.

It relies on the postulate that the dynamics of the temporal POD coefficients $a^{(n)}(t)$ is driven by a set of ordinary differential equations (ODEs) of the same nature as those that can be obtained via a POD-Galerkin approach, namely, polynomial ODEs of quadratic order

$$
\dot{a}^{(i)}=D_{i}+L_{i j} a^{(j)}+Q_{i j k} a^{(j)} a^{(k)},
$$

with implicit summation over repeated indices, $i \in\left[1: N_{t r}\right]$ and $1 \leqslant i \leqslant j \leqslant k \leqslant N_{t r}, N_{t r}$ being the number of POD modes retained to develop the LODS. In this approach, $D_{i}, L_{i j}$, and $Q_{i j k}$ are the unknown parameters that must be indentified. From the DT-SPIV database, $N_{s}$ pairs of POD coefficients $\left\{a^{(n)}(t), a^{(n)}(t+\tau)\right\}$ can be computed by solving the eigenvalue problem (1). Thus, time derivatives $\dot{a}_{n}(t)$ of POD coefficients $a^{(n)}(t)$ can be computed by a second order finite difference scheme between two samples separated by a time delay $\tau$,

$$
\dot{a}^{(n)}(t+\tau / 2)=\left(a^{(n)}(t+\tau)-a^{(n)}(t)\right) / \tau .
$$

To ensure that the samples of $\dot{a}^{(n)}$ and $a^{(n)}$ are evaluated at the same instant $t$, temporal coefficients are evaluated as $a^{(n)}(t+\tau / 2)=\left(a^{(n)}(t+\tau)+a^{(n)}(t)\right) / 2 .{ }^{14}$ From the $N_{s}$ samples of both $a^{(n)}(t)$ and $\dot{a}^{(n)}(t)$, the LODS parameters $D, L$, and $Q$ can be estimated by a least-squares approach. Once these parameters are obtained, a fourth order Runge-Kutta algorithm is used to integrate in time the system of ODES (9) in order to compute the temporal evolution of modeled eigenmodes $\widetilde{a}^{(n)}(t)$.

Application. The above-presented method for LODS identification from experimental databases ${ }^{14}$ is applied here to build a quadratic dynamical system modeling the spatio temporal dynamics of the mixing layer in a cross section of the flow.

As the LODS parameters are determined from experimental data, their estimation is affected by the presence of measurement noise in the SPIV velocity fields. As a matter of fact, measurement noise, which is due to subpixel estimation error, peak-locking bias, and error induced by stereoscopic reconstruction algorithms, is a priori space and time dependent. Moreover, given the local character of the PIV processing of the particle images, the spatial scale of this noise can be considered as low but nonzero. The two-point spatial correlation tensor is thus also affected by the measurement uncertainty. Consequently, both the energy levels (e.g., POD eigenvalues $\lambda^{(n)}$ ) and the shapes of the eigenvectors $\phi^{(n)}$ are influenced.

Study of the energetic content of the POD eigenvalues obtained for both velocity fields $\mathbf{u}^{A}(y, z, t)$ and $\mathbf{u}^{B}(y, z, t)$ 
(a)

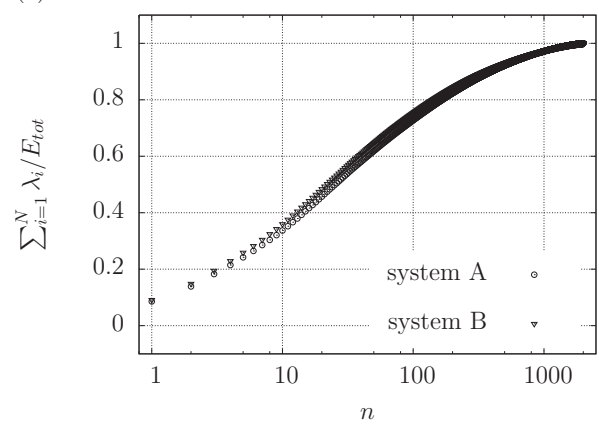

(b)

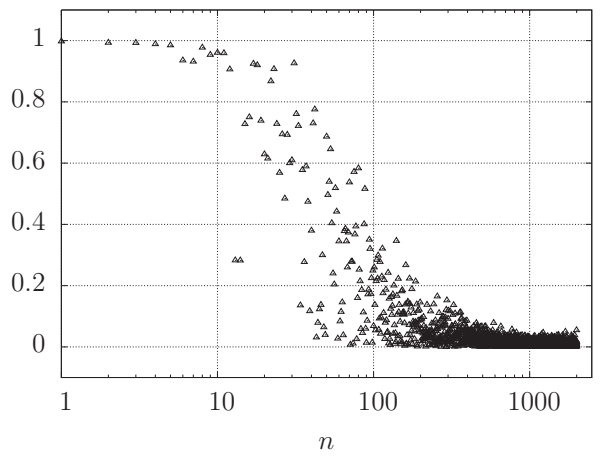

FIG. 2. (Top) Energetic convergence of the eigenvalues from the POD decomposition of the PIV velocity fields. (Bottom) Inner product between POD modes of the same order obtained from both PIV systems $A$ and $B$ as a function of the mode order $N$.

measured by the SPIV systems $A$ and $B$, respectively, does not reveal any significant discrepancy [Fig. 2(a)]. Evaluation of the noise influence on the POD decomposition is then performed by comparing the POD modes obtained from the two sets of velocity fields. This comparison is performed through the computation of the inner product between two modes of the same order, $\phi_{A}^{(n)}(y, z)$ and $\phi_{B}^{(n)}(y, z)$, issued from the velocity fields $\mathbf{u}^{A}(y, z, t)$ and $\mathbf{u}^{B}(y, z, t)$, respectively. This inner product value provides a global information relative to the modes as it is obtained via spatial integration and takes into account the three velocity components. In the absence of measurement noise, these POD modes should be perfectly identical, leading to an inner product value of 1 . Any departure from the value of 1 is characteristic of discrepancies between the two modes. The value of this inner product obtained for all the POD modes is shown in Fig. 2(b). It is comprised between 0.9 and 1 for the first 12 modes and then decreases for increasing mode order. Thus, the higher the mode order, the more significant the influence of the measurement noise. Consequently, $N_{t r}=12$ POD modes are retained to develop the LODS, representing almost $40 \%$ of the total turbulent kinetic energy in the whole section [Fig. 2(a)]. This amount of turbulent kinetic energy turned out to be sufficient to model the large scale structures of the mixing layer. ${ }^{16}$ It must be mentioned here that different tests were conducted in order to try to build LODS comprising more POD modes in order to improve the dynamical richness of the modeled flow. However, in spite of the use of the stabilization method detailed below, it turned out that it was impossible to derive from the experimental database a stable LODS containing more than 12 POD modes, confirming the influence of the measurement uncertainty.

Once the parameters $D, L$, and $Q$ of Eq. (9) are indentified, the 12-equation model is integrated, using one sample $\left\{a^{(n)}(t), a^{(n)}(t+\tau)\right\}$ from the database as initial condition in order to obtain time-varying modeled eigenmodes $\widetilde{a}^{(n)}(t)$. Velocity fields associated with the coherent structures are then reconstructed as the linear combination of the spatial modes $\phi^{(n)}(y, z)$ and the coefficients $\widetilde{a}^{(n)}(t), 1 \leqslant n \leqslant N_{t r}$. As only the first 12 modes are retained in the model, the interaction with modes of higher order is not represented. In order to compensate for the absence of energy transfer toward the small scales, an artificial viscosity is added in the model by amplifying the linear term $L_{i j}$. This artificially introduced linear damping, which aims at modeling the interaction between the used POD modes and the other modes rejected from the LODS, has been used to derive stable LODSs in various flow configurations such as wall-bounded flows, ${ }^{24}$ mixing layer flows, ${ }^{25}$ or flow resulting from the interaction of a turbulent mixing layer and the wake of a cylinder. ${ }^{26}$ From these studies, it appears that no systematic method has been yet developed to provide the correct value of this stabilizing parameter. The stabilization of the model thus relies on a trial and error procedure to find the correct value for the damping, which depends on several parameters such as the flow configuration and its complexity or the number of retained POD modes. In the present case, stability is obtained for amplification factors greater than 1.765 , value determined by a parametric study. ${ }^{16}$ This value is kept throughout the study.

The vorticity modulus of the resulting velocity fields is represented in Fig. 3(a) in a convected frame of reference using the transformation $x=-U_{m} t$. Visual realistic behavior of the flow motion is exhibited, with spanwise structures and 3D character. Figures 4 and 5 show comparisons of the statistics of the velocity fields obtained from the 12-mode LODS with those from the unfiltered experimental data. These comparisons are provided to show what is the contribution of the model to the original flow and to verify that this contribution corresponds well to the coherent structures that it is supposed to model. The spatial distribution of turbulent kinetic energy contained in the coherent part modeled by the 12-mode LODS is shown in Fig. 4. This distribution corresponds well to the presence of turbulent structures located on average on the axis of the shear layer with almost zero energy in the outer region. The difference of level between the total turbulent energy and the energy contained by the 12 mode modeled flow is directly due to the fact that only 12 POD modes are retained to develop the LODS. However this lower level of energy is believed to be sufficient to achieve the modeling of the dynamics of the coherent structures. In their testing of several coherent structure eduction methods, Bonnet et $a .^{27}$ showed that, whatever the retained method, the contribution of the coherent part of the flow to the energy is of the order of $50 \%$. Moreover, as detailed below, the analysis of the velocity spectra corroborates the fact that the LODS reproduces the correct turbulent scales. Spectra of the three velocity components of the coherent part modeled by the 12-mode LODS obtained on the mixing layer axis are 
(a)

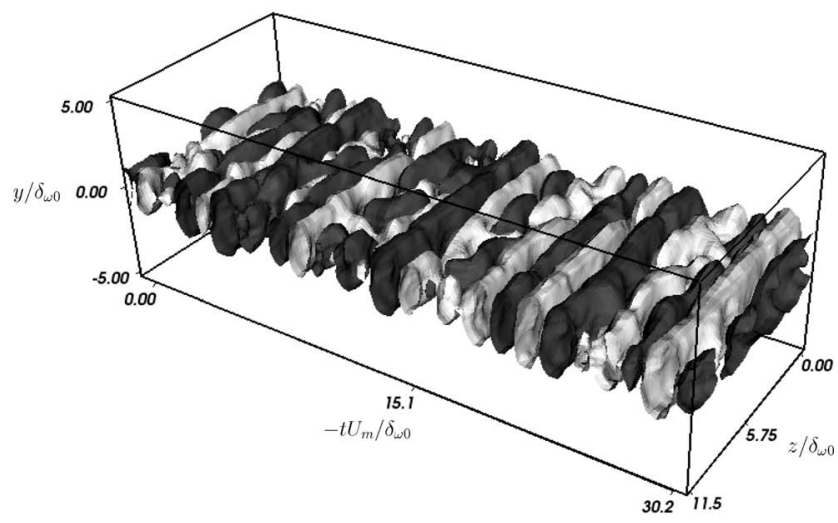

(b)

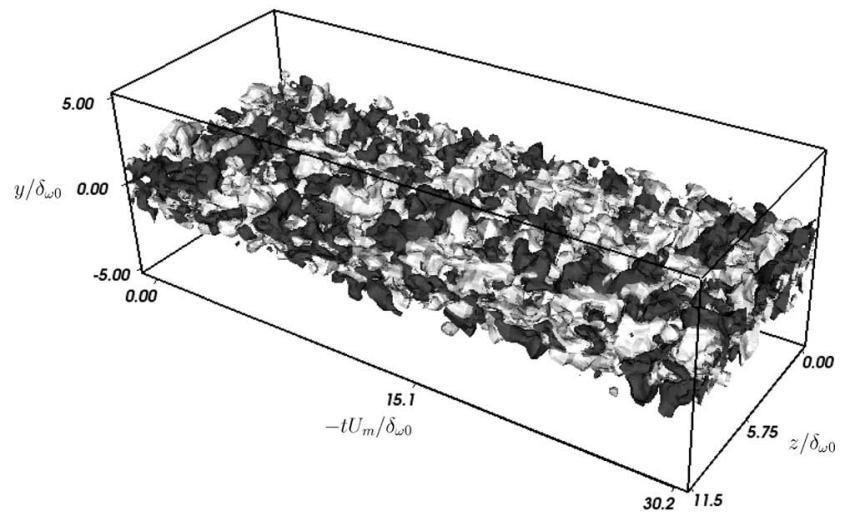

(c)

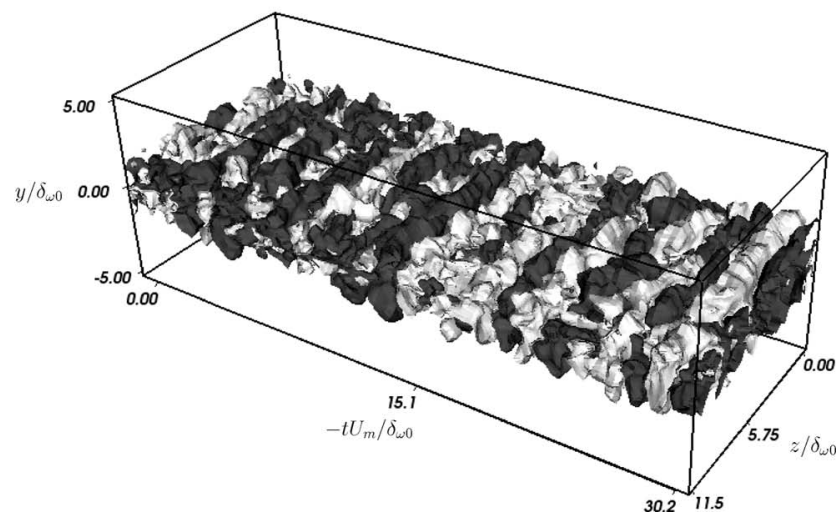

FIG. 3. Time evolution of isosurfaces of the instantaneous vertical velocity component of (a) the flow modeled by the 12-mode low-order model, (b) the small-scale motion modeled by random number time series, (c) the flow used as inflow condition resulting of the combination of (a) and (b) via Eq. (8) (dark gray: upward velocity; light gray: downward velocity).

presented in Fig. 5 and are compared to spectra measured at the same location with hot wires. As expected, the lowfrequency range is well reproduced by the LODS, most of the energy being contained in frequencies below $f \delta_{\omega 0} / U_{m}$ $=0.7$. Moreover, frequencies characteristic of the KelvinHelmholtz structures, corresponding to a peak in the experimental spectra around frequencies of order $f \delta_{\omega 0} / U_{m}=0.3,{ }^{19}$ are also well reproduced for the three components. It should be noted here that, as only 12 POD modes are retained, the

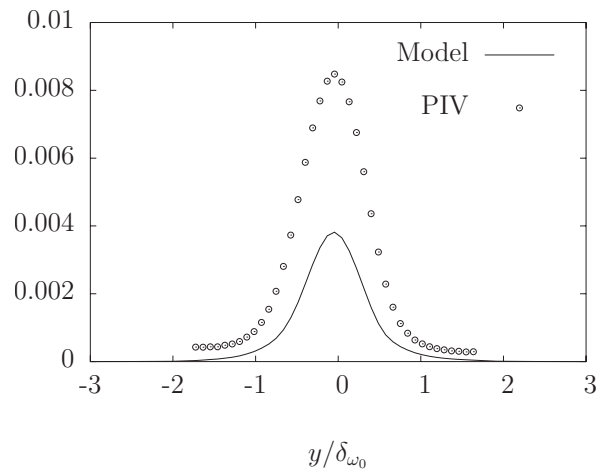

FIG. 4. Distribution of the turbulent kinetic energy (symbols) directly measured by PIV and (line) reproduced by the 12-mode low-order model.

frequency content of the modeled flow is not as rich as the the one measured by hot wire. Moreover, the energy is not equally distributed among the three velocity components, the modeled longitudinal $u$ and transversal $w$ velocity compo-

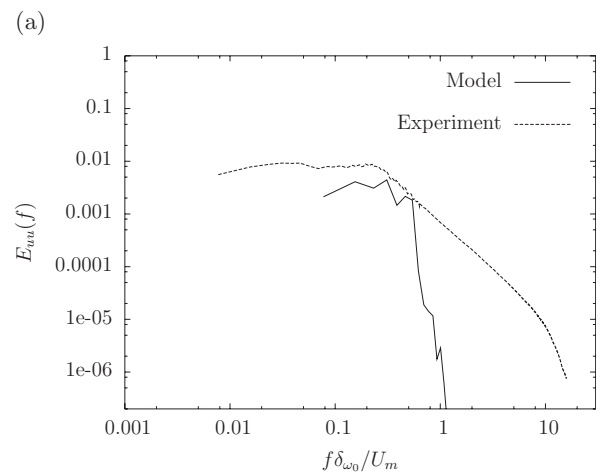

(b)

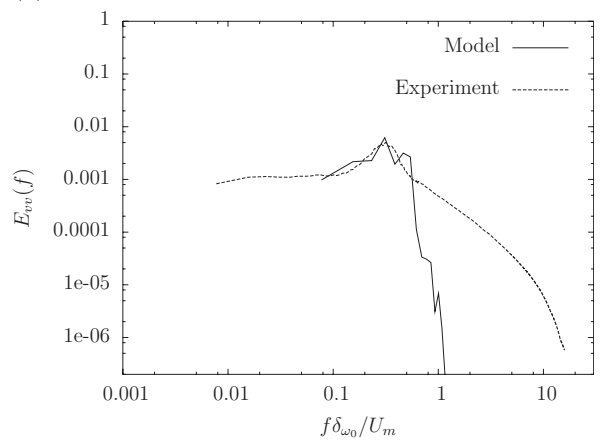

(c)

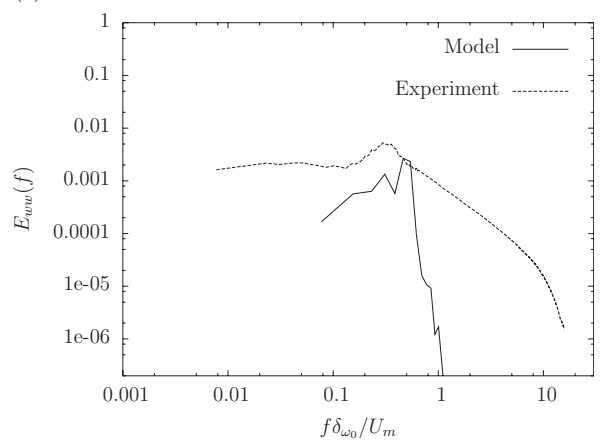

FIG. 5. Energy spectrum of (a) the longitudinal, (b) the vertical, and (c) the spanwise velocity components on the shear-layer axis obtained from (solid line) the 12-mode model; and (dashed line) from hot-wire measurements. 
nents presenting a lack of energy when compared to the experimental measurements. This can be explained by the strong energetic signature of the vertical component in the considered frequency range ${ }^{19}$ and also by the fact that the energy equipartition among the three velocity components is not ensured by the POD formalism. This spectral analysis confirms that the behaviour of the large-scale structure can correctly be modeled by a LODS built from a database containing only uncorrelated samples of velocity and acceleration fields.

\section{Smaller scale dynamics modeling}

In order to represent the total amount of turbulent kinetic energy of the flow, it is necessary to take into account the higher order POD modes not retained in the LODS previously described. This is achieved via the modeling of the temporal coefficients $b^{(n)}(t)$ in Eq. (8). As proposed by Perret et al. ${ }^{13}$ time series of random numbers are employed for that purpose. $N_{s}-N_{t r}$ independent Gaussian time series of random numbers $g^{(n)}(t)$ are generated first. These series have zero mean, unity variance and are, by construction, mutually uncorrelated in time. In order to ensure that the reconstructed velocity fields present a spectral content representative of a turbulent flow, a spectral transfer function designed to impose a modified Vòn Kàrman spectrum $E_{\text {ref }}(f)$ of the same form as the one proposed by $\mathrm{PaO}^{28}$ is applied,

$$
\hat{b}^{(n)}(f)=\hat{g}^{(n)}(f)\left(\frac{E_{\mathrm{ref}}(f)}{E_{g g}(f)},\right)^{1 / 2},
$$

where $\hat{g}^{(n)}(f)$ is the Fourier transform of $g^{(n)}(t)$ and $E_{g g}(f)$ its spectrum. The reference spectrum is of the form ${ }^{28}$

$$
E_{\text {ref }}(f)=\frac{C}{f_{L}}\left[1+\left(f / f_{L}\right)^{2}\right]^{-5 / 6} \exp \left[-2\left(f / f_{d}\right)^{2}\right]
$$

where $f_{L}$ and $f_{d}$ are the integral and dissipation scales, respectively. These two parameters are evaluated from previous hot-wire velocity measurements to ensure that the modeled POD modes present a correct spectral behavior. Eventually, the coefficients $b^{(n)}(t)$ are normalized so that $\left\langle b^{(n)}(t) b^{(m)}(t)\right\rangle=\lambda^{(n)} \delta_{m}^{n}$ in order to ensure that each mode contains the correct energy. Thus, generated time series satisfy all the properties of the real POD coefficients, namely, having zero mean, being mutually uncorrelated in time, and having an energy equal to the corresponding eigenvalue. They also present a realistic turbulent spectral content. Consequently, reconstructing velocity fields as linear combination of these temporal coefficients and the original spatial POD eigenvectors ensures that the reconstructed data share many of the statistical properties of the original data, e.g., one- and two-point spatial statistics and a realistic temporal spectrum. However, as shown in Fig. 3(b), a visualization of the corresponding velocity field, represented in a convected frame of reference using the transformation $x=-U_{m} t$, does not exhibit any particular spatial organization, resulting from the random character of the generated $b^{(n)}(t)$.

\section{Spatial interpolation-extrapolation}

The second part of the experiment-computation interfacing process consists in both the extrapolation and the interpolation of the experimental data onto the grid of the inlet section. The generated velocity fields must present the same spatial resolution as the computation and must satisfy, at each time step, all the boundary conditions retained to perform the calculation. In the present case, given the geometry of the mixing layer flow, the simulation is performed with a periodic condition in the homogeneous spanwise direction and a free-slip condition on the horizontal boundaries. Thus, the generated velocity fields have to be interpolated and extrapolated in both the vertical and spanwise directions and must satisfy the boundary conditions retained for the computation. The procedure developed by Perret et al., ${ }^{13}$ based on the processing of the spatial snapshot POD eigenvectors, is applied here.

\section{Homogeneous direction processing}

Imposing a periodic character in the spanwise direction on the experimental data that are a priori nonperiodic is performed in two steps. First, the minimum size $L_{z}$ of the domain on which the periodic condition must be enforced is determined by using the fact that POD modes degenerate into harmonic modes when a homogeneous direction is considered. ${ }^{21}$ Following the work of Coiffet et al. ${ }^{29}$ the POD can be used to detect a spanwise wavelength in the set of data. These authors demonstrated that POD modes obtained in a homogeneous direction are very close to harmonic modes and that their shapes are not affected by the finite size of measurement domain as harmonic modes issuing from a Fourier analysis would be. In the present case, a onedimensional POD is performed in the spanwise direction $z$ at each vertical position $y$ by decomposing the two-point velocity correlation tensor $R_{i i}\left(y, z, z^{\prime}\right)$. Each obtained onedimensional POD mode is then approximated by a sine function that gives access to its wavelength. The spanwise extent $L_{z}$ is then chosen to be equal or close to a multiple of the spatial wavelength of the POD modes computed in the spanwise direction. In the present study, the obtained $L_{z}$ is 1.83 times larger than the spanwise extent of the original measurement domain.

Second, on the basis of the previous data analysis, the velocity fields are extrapolated and rendered periodic in the spanwise direction through the processing of their twodimensional snapshot POD modes. To perform this spanwise extrapolation, the selective deconvolution method is employed here. This technique was proposed by Franke ${ }^{30}$ to extrapolate signals, the spectrum of which is band limited and presents energy dominant frequencies. The convergence and the uniqueness of the solution of this iterative algorithm based on direct and inverse FFT was proved by Van Droogenbroeck. ${ }^{31}$ This method enables both the extrapolation and the periodicity prescription at the same time, while removing the windowing effect involved in spectral analysis when limited measurement domain sizes are considered. Given the fact that the snapshot POD modes are representative of the turbulent structures contained in the flow and that 

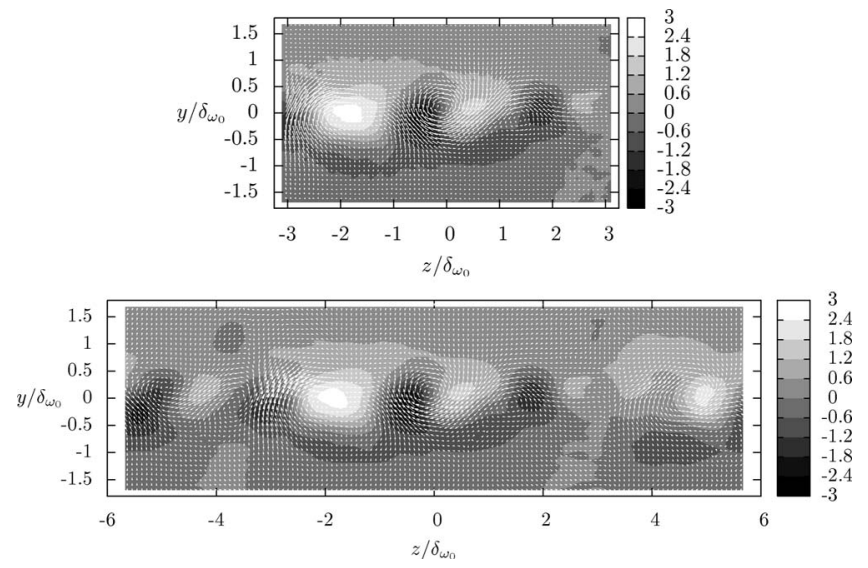

FIG. 6. Example of a snapshot POD eigenvector $\phi_{i}^{(n)}(y, z)$ (top) and its spanwise extrapolation (bottom) for $n=8$ (vectors: in-plane velocity; colors: longitudinal velocity).

a preferred wavelength is known in the spanwise homogeneous derection, the conditions to apply Franke's algorithn are satisfied. Thus, selective deconvolution is applied here via bidimensional FFT in order to spread the motives corresponding to the turbulent structures embedded within the flow. Figure 6 shows an example of a snapshot POD mode $\phi_{i}^{(n)}(y, z)$ and its spanwise extrapolation. It can be noticed that the applied procedure ensures the spanwise periodicity of the mode and does not introduce any spatial discontinuities.

\section{Extrapolation in the inhomogeneous direction}

Given the limited extent of the measured velocity fields in the $y$-direction, vertical extrapolation is required to prevent the computed flow from being confined. Furthermore, a free-slip condition must be enforced to the generated inflow conditions. For that purpose, the method developed by Druault et al. ${ }^{12}$ and applied on SPIV measurements by Perret et al. ${ }^{13}$ is employed here.

The vertical processing of the velocity fields is based on a one-dimensional POD approach to extrapolate the bidimensional snapshot POD modes $\phi^{(n)}(y, z)$ in the $y$-direction at each $z$-location independently. In a first step, the two-point correlation tensor $R_{i i}\left(y, y^{\prime}\right)=\left\langle u_{i}(y, t) u_{i}\left(y^{\prime}, t\right)\right\rangle$ is computed from the original database, where $\langle\cdot\rangle$ denotes here the averaging operator in both temporal and spanwise directions.

A POD decomposition of $R_{i i}\left(y, y^{\prime}\right)$ is performed to obtain $N_{y}$ one-dimensional eigenvectors $\psi_{i}^{(m)}(y), N_{y}$ being the number of points in the vertical direction. These vectors forming a basis for the flow fields, they are also a basis onto which the snapshot POD modes $\phi_{i}^{(m)}(y, z)$ can be decomposed at each spanwise location,

$$
\phi_{i}^{(n)}(y, z)=\sum_{m=1}^{N_{y}} p^{(m, n)}(z) \psi_{i}^{(m)}(y),
$$

$p^{(m, n)}(z)$ being the projection coefficient of the bidimensional POD mode $\phi_{i}^{(n)}(y, z)$ onto the $m$ th one-dimensional mode at the spanwise position $z$.
In a second step, as proposed by Druault and Delville, ${ }^{32}$ the tensor $R_{i j}$ can be extrapolated on the numerical mesh by using the fact that, in free-shear flows, the POD modes, in the same manner as the turbulent kinetic energy, must decrease to zero in the inhomogeneous direction. Moreover, in the case of the mixing layer, these authors pointed out that the decay of the POD modes is exponential when $y$ tends to infinity. Thus, $\psi_{i}^{(n)}(y)$ are extrapolated into new onedimensional modes $\widetilde{\psi}_{i}^{(n)}(y)$ in a larger domain.

New extrapolated bidimensional POD modes $\widetilde{\phi}_{i}^{(n)}(y, z)$ can finally be reconstructed on the larger computational domain with the modes $\tilde{\psi}_{i}^{(n)}(y)$ and the previously computed projection coefficients $p^{(m, n)}(z)$,

$$
\widetilde{\phi}_{i}^{(n)}(y, z)=\sum_{m=1}^{N_{y}} p^{(m, n)}(z) \widetilde{\psi}_{i}^{(m)}(y) .
$$

These new snapshot POD modes can now serve as a basis to rebuild instantaneous velocity fields that are periodic in the spanwise direction and extrapolated on a larger domain corresponding to the computational domain. The final generated velocity fields $\widetilde{u}_{i}(y, z, t)$ will then read

$\widetilde{u}_{i}(y, z, t)=\sum_{n=1}^{N_{t r}} \widetilde{a}^{(n)}(t) \widetilde{\phi}_{i}^{(n)}(y, z)+\sum_{n=N_{t r}+1}^{N_{s}} b^{(n)}(t) \widetilde{\phi}_{i}^{(n)}(y, z)$.

\section{GENERATED VELOCITY FIELDS}

The above-presented strategy has been employed to generate unsteady velocity fields from the DT-SPIV measurements performed with the experimental setup presented in Sec. II. A synthetic database of 20000 time steps with a temporal sampling frequency (which is equivalent to a physical data acquisition frequency of $130 \mathrm{kHz}$ ) in agreement with the computational time step has been created.

Time evolution of the vertical component of the velocity field generated with the present strategy is presented in Fig. 3 (c) in a convected frame. The generated flow exhibits a highly complex 3D organization resulting from the linear combination of large structures [Fig. 3(a)] and more random small-scale motions [Fig. 3(b)]. Moreover, no spatial discontinuities are noticeable in the spanwise direction, confirming the performance of the proposed extrapolation procedure.

Distributions of the Reynolds stresses obtained in the vertical direction after integration over the spanwise extent are presented in Fig. 7. Agreement is obtained with the experimental data as concerns both energy level and spatial distribution across the shear layer. The anisotropy of the flow is correctly reproduced by the synthesized data.

Total velocity spectra [Figs. 8(a)-8(c)] computed at the center of the mixing layer are compared to hot-wire measurements performed at the same location $(x=300 \mathrm{~mm}, y=0)$. Spectra of the modeled data now show a wide-range spectral content in agreement with the experiment. Moreover, dominant frequencies, typical of the spatiotemporal organization of the mixing layer, are present. The spectrum of the total turbulent kinetic energy of the modeled data [Fig. 8(d)] compares well with the experimental data, both in terms of shape 


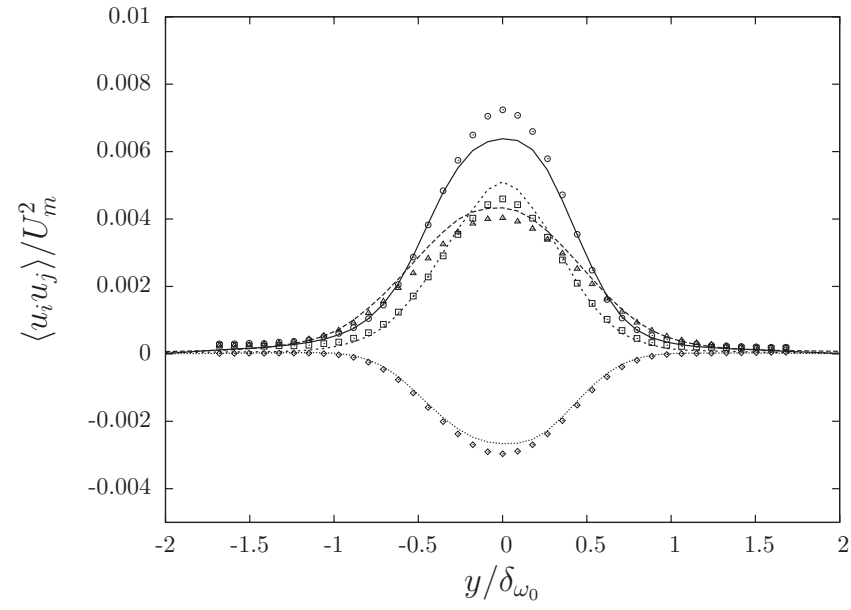

FIG. 7. Distribution of the Reynolds stresses (lines) of the generated inflow velocity fields and (symbols) directly measured by PIV. Open circles: $\left\langle u^{\prime 2}\right\rangle$; open triangles: $\left\langle v^{\prime 2}\right\rangle$; open squares: $\left\langle w^{\prime 2}\right\rangle$; open diamonds: $\left\langle u^{\prime} v^{\prime}\right\rangle$.

and levels. However, it appears that the energy content of these dominant frequencies, corresponding to the spectral content of the LODS, is slightly overestimated. This may be explained by the fact that the temporal dynamics of the velocity field is given by the combination of the $b^{(n)}(t)$ modes [see Eq. (8)] that are modeled by synthetic signals having a wide-range energy spectrum, and the $\widetilde{a}^{(n)}(t)$ modes resulting from the integration of the 12-mode LODS that have a poorer frequency content. The low-frequency range (below

(a)

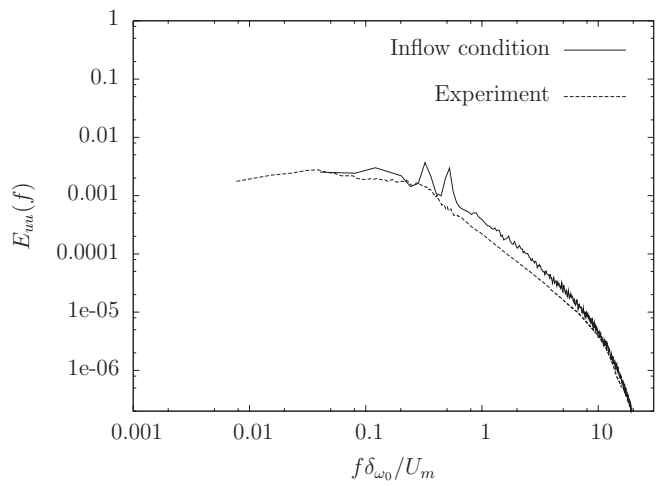

(c)

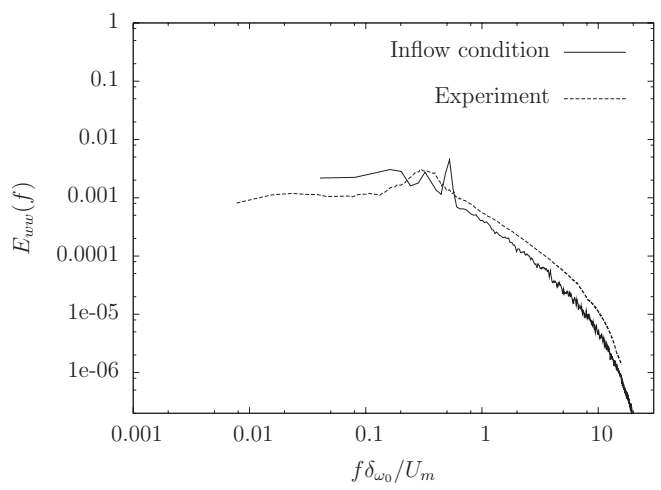

$\left.f \delta_{\omega 0} / U_{m}=0.7\right)$ is thus increased, most of the energy modeled by the LODS being contained in this frequency domain. On the contrary, higher-frequency energy is mainly due to the contribution of the $b^{(n)}(t)$ 's for which a simple model spectrum is prescribed, given the fact that no other information is available from the SPIV measurements.

Figure 9 presents two-point spanwise correlations obtained at various locations across the mixing layer, with comparison to SPIV results. Correlations from experimental data are directly computed by $R_{i i}\left(y, \delta_{z}\right)=\left\langle u_{i}(y, z, z) u_{i}\left(y, z, z+\delta_{z}\right)\right\rangle$ (the operator $\langle\cdot\rangle$ denoting here the average operator in both temporal and spanwise directions), whereas correlations obtained from generated flow fields are computed via Fourier transform of the periodic synthetic velocity fields. Only half of the spanwise extent of the computational domain is shown here, the spanwise correlation tensor being symmetric with respect to the vertical axis, given the homogeneity of the spanwise direction. It should be noted here that long-range correlation levels are slightly overestimated near the center of the mixing layer $\left(y / \delta_{\omega 0}=-0.2,0.0,+0.2\right)$. This may result from a bias introduced by the selective deconvolution algorithm in the low-frequency part of the spatial spectrum of the velocity field. This bias leads to a slight alteration of the larger spanwise wavelengths but does not affect the shortrange correlation for which the higher correlation levels are obtained. Thus, it can be admitted that the spanwise organization of the flow is correctly reproduced by the extrapolated flow fields. In particular, streamwise vortices, the statistical

(b)

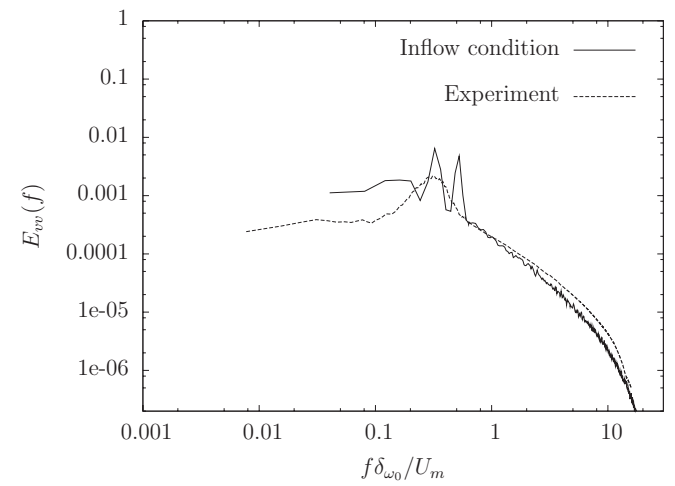

(d)

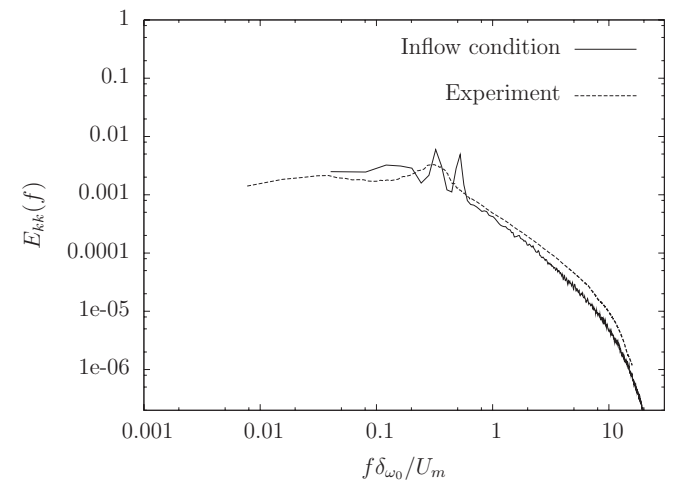

FIG. 8. Energy spectrum of (a) the longitudinal, (b) the vertical, (c) the spanwise velocity components, and (d) the total turbulent kinetic energy on the shear-layer axis of (solid line) the generated inflow data and (dashed line) from hot-wire measurements. 

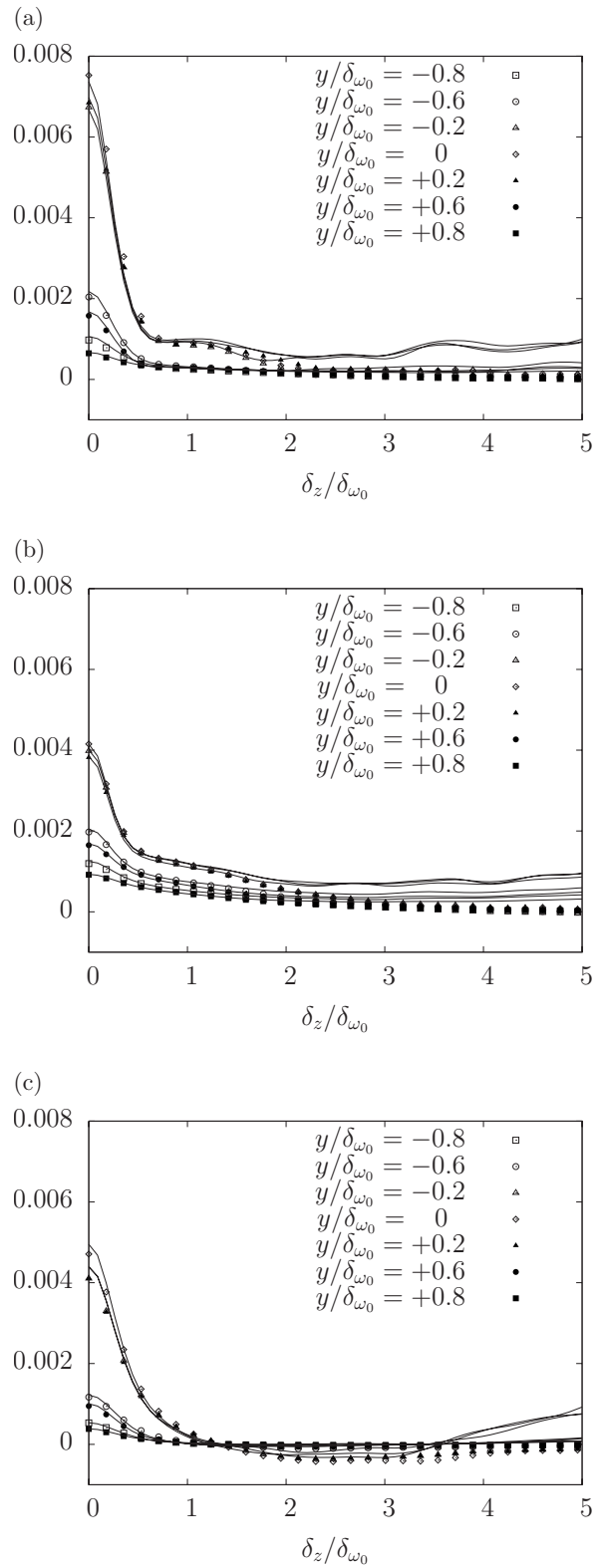

FIG. 9. Two-point spanwise correlation $R_{i i}\left(y, \delta_{z}\right)$ of the (a) longitudinal, (b) vertical, and (c) spanwise velocity components evaluated at various positions across the mixing layer (symbols: measurements; lines: generated data).

footprint of which is the shape of the correlation at short distance, are not altered by the developed extrapolation procedure.

Comparisons between spatiotemporal correlation coefficients obtained for a reference point located in the center of the shear layer from the generated velocity fields and from hot-wire measurements are presented in Fig. 10. In both cases, correlations are computed via Fourier transform of velocity time series. Correlations computed from modeled data compare well with the statistics evaluated from hot-wire measurements. It should be noted here that the integral scales in the temporal direction are slightly underestimated in the synthesized velocity fields. This is attributed to the fact that the experimental velocity spectra are not perfectly reproduced, given the frequency content of the coherent part mod- (a) $R_{u u}\left(y, y^{\prime}, \tau\right)$

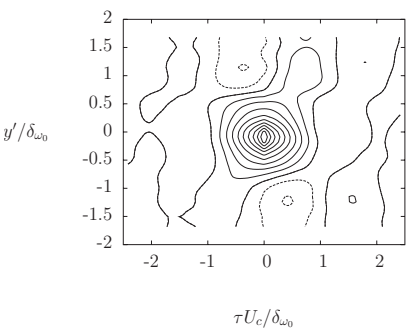

(c) $R_{v v}\left(y, y^{\prime}, \tau\right)$

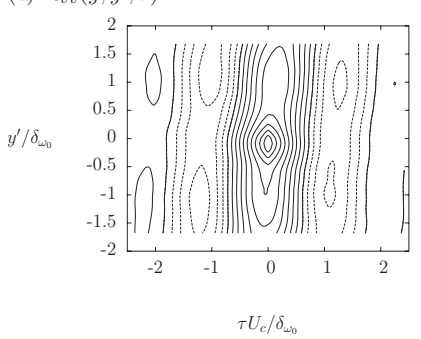

(e) $R_{w w}\left(y, y^{\prime}, \tau\right)$

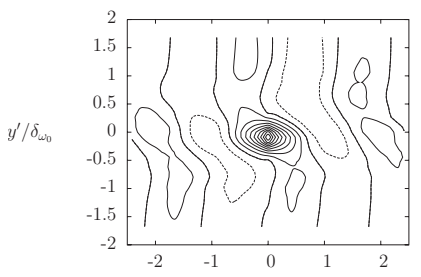

$\tau U_{c} / \delta_{\omega_{0}}$ (b) $R_{u u}\left(y, y^{\prime}, \tau\right)$

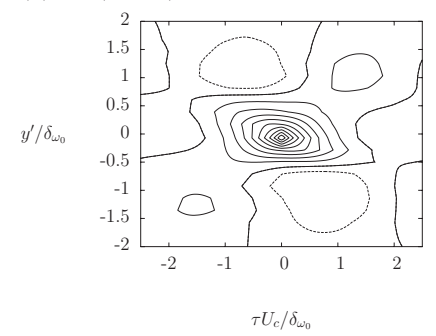

(d) $R_{v v}\left(y, y^{\prime}, \tau\right)$

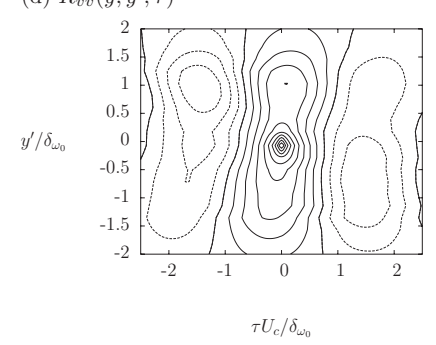

(f) $R_{w w}\left(y, y^{\prime}, \tau\right)$

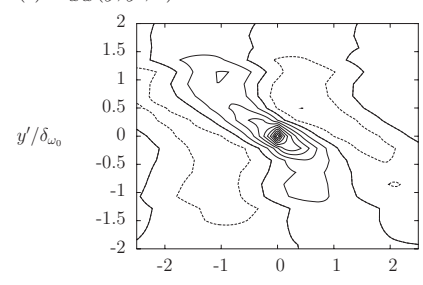

$\tau U_{c} / \delta_{\omega_{0}}$
FIG. 10. Spatiotemporal two-point correlation coefficient $R_{i i}\left(y, y^{\prime}, \tau\right)$ evaluated in the center of the mixing layer $\left(y=0, x=x_{0}\right)$ from (left) the generated inflow conditions and (right) directly from hot-wire measurements: (solid) positive contours in increment of 0.1 and (dashed) negative contours in increment of 0.1 .

elled by the 12-mode LODS. However, shapes of the obtained correlations are well reproduced, especially the inclination and alternance of the region of negative correlation, typical of the spatiotemporal organization of mixing layer flows.

Instantaneous flow visualizations as well as one- and two-point statistics in space and time presented in this section confirm the ability of the present method to generate, from a SPIV experimental database that does not contain any time series, synthetic flow fields that have a realistic spatiotemporal organization in agreement with the targeted flow.

\section{LES OF A TURBULENT MIXING LAYER USING THE GENERATED INFLOW CONDITIONS}

In order to validate the proposed inflow data generation method, a LES of a plane turbulent mixing layer has been performed. To enable direct comparison with hot-wire measured data, all the parameters of the simulated flow were chosen to match those of the real flow. The presented simulation thus deals with a high Reynolds number turbulent mixing layer, the inlet section of which corresponds to the beginning of the self-similarity region of the mean flow. 


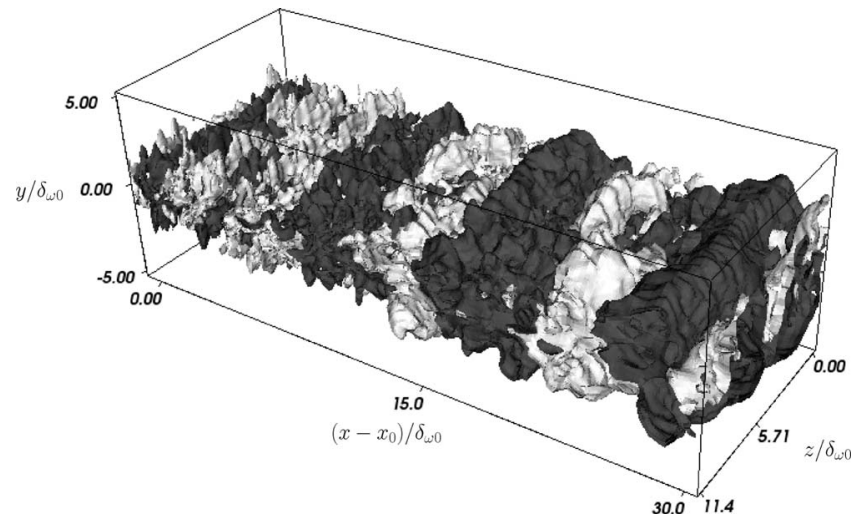

FIG. 11. Isosurface of the instantaneous vertical velocity component computed from the present LES.

\section{A. Numerical method}

The present LES was performed with CODE_SATURNE, a finite volume solver on unstructured grids developed at EDF, for vectorial and parallel computing. ${ }^{33}$ The filtered, incompressible Navier-Stokes equations are solved with a dynamical Smagorinsky subgrid scale model. Space discretization is based on a collocation of all the variables at the center of gravity of the cells, using central differencing. Time discretization is based on the Crank-Nicolson/Adams-Bashforth method. The velocity/pressure system is solved by a SIMPLEC algorithm, with the Rhie and Chow interpolation. The Poisson equation is solved using a conjugate gradient method, with diagonal preconditioning. Computations are performed using eight processors of a personal computer cluster. The inlet of the computational domain corresponds to the measurement section and the above-presented generated data are used as inlet conditions. The Reynolds number in the inlet section of the computation corresponds to the experimental one, namely, 36000 . The spatial extent of the domain is $L_{x} \times L_{y} \times L_{z}=30 \delta_{\omega 0} \times 10 \delta_{\omega 0} \times 11 \delta_{\omega 0}$, meshed nonregularly with $N_{x} \times N_{y} \times N_{z}=224 \times 53 \times 129$ points. It should be noted here that the spatial resolution retained in the inlet section of the simulation corresponds exactly to that of the SPIV experiment. As a consequence, there is no difference between the spatial scales of the generated velocity fields and those simulated at the entry of the computational domain. The streamwise extent of the computational domain is chosen to enable the simulation of a significative evolution of the flow, corresponding to the doubling of the shear-layer thickness $\left[\left(x-x_{0}\right) / \delta_{\omega 0} \leqslant 23\right]$. The last part of the domain is used to avoid some possible influence of the outflow condition.

\section{B. LES results}

Study of an instantaneous vertical velocity component field of the simulated flow (Fig. 11) reveals that highly complex 3D turbulent flow structures are present downstream of the inlet section. On such a visualization, no region of transition or adjustment can be seen at the beginning of the computational domain.

To further and more quantitatively characterize the LES (a)
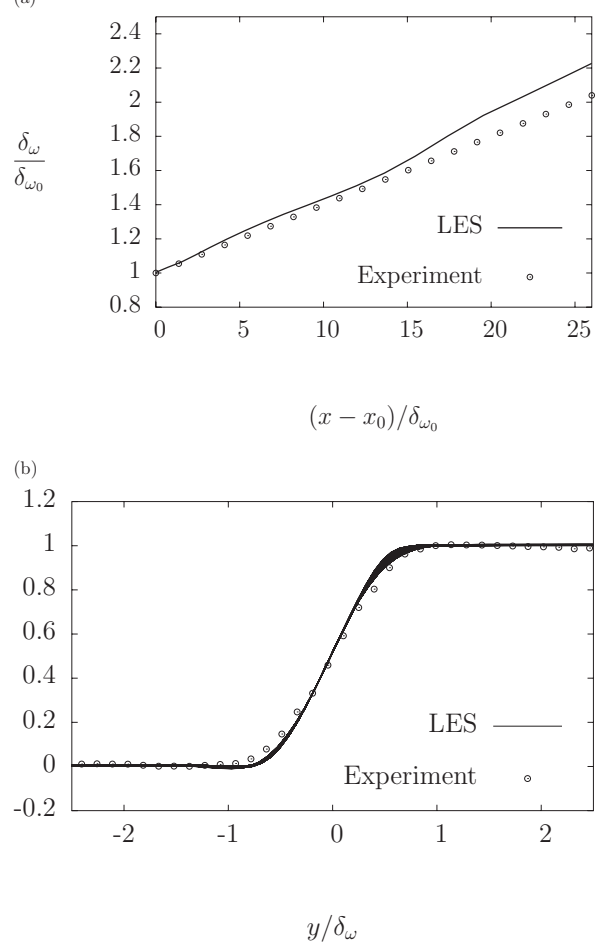

FIG. 12. Longitudinal evolution of (top) the vorticity thickness of the mixing layer and (bottom) the profiles of mean longitudinal velocity component (86 profiles superimposed); lines: computation; symbols: experiment.

results, mean flow characteristics are first analyzed and compared to the results obtained via Pitot tube measurements by Perret. ${ }^{16}$ Figure 12(a) presents the downstream evolution of the vorticity thickness $\delta_{\omega}$. It reveals that the growth rate of the simulated flow is in good agreement with the experimental one. Moreover, neither modification nor adjustment of the growth rate near the inlet region can be seen, as it is generally the case for different methods of inlet condition generation, ${ }^{12}$ confirming that the inflow data are well adapted. This is confirmed by the study of the mean longitudinal velocity profiles that superimpose well when plotted in self-similar coordinates [Fig. 12(b)].

Figure 13 presents the longitudinal evolution of the profiles of both the turbulent kinetic energy and the Reynolds shear stress. Results of the present LES are compared to hot-wire measurements and LES results obtained by Perret et al. ${ }^{13}$ using inflow conditions modeled by time series of random numbers with prescribed spectra (e.g., without any dynamical system to account for the presence of the large scale structures). When compared to experimental results, the turbulent kinetic energy and Reynolds shear stress [Figs. 13(a) and 13(b)] are not subject to a strong adjustment downstream of the inlet section. Turbulent kinetic energy is slightly underestimated in the upstream part of the domain but does not drop dramatically as it can occur when less realistic inlet conditions are employed. ${ }^{10,12}$ The same behavior is obtained for the Reynolds shear stress. Moreover, when plotted in self-similar coordinates, these profiles confirm the correct spreading of the shear layer as it evolves further downstream.

Figure 14 shows additional comparisons of the longitu- 
(a)

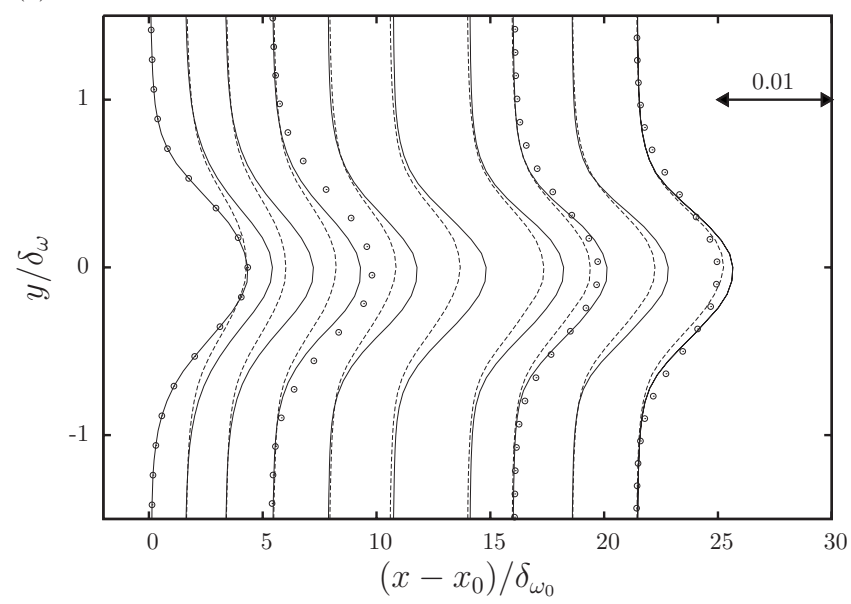

(b)

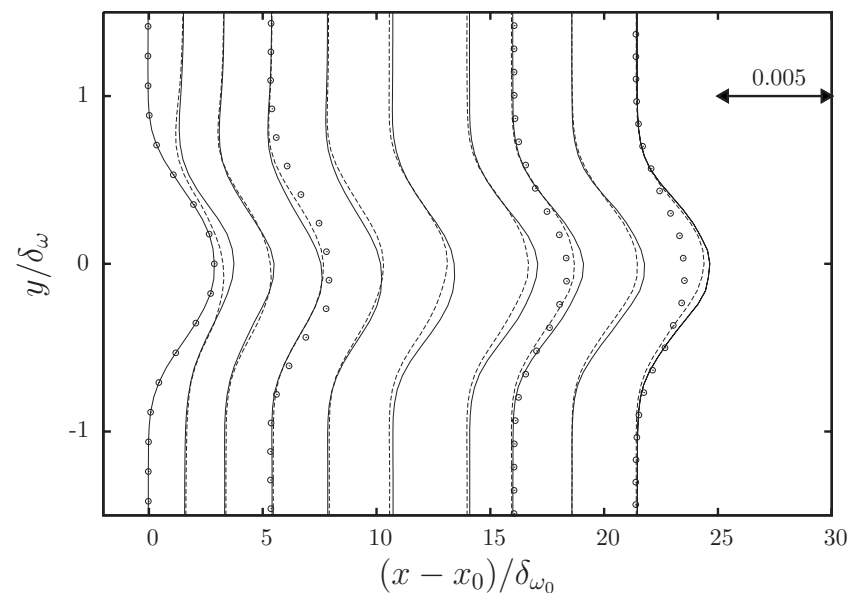

FIG. 13. Longitudinal evolution of (top) the turbulent kinetic energy $\langle k\rangle / U_{m}^{2}$ and (bottom) the shear stress $-\left\langle u^{\prime} v^{\prime}\right\rangle / U_{m}^{2}$; solid lines: present computation; dashed lines: LES of Perret et al. (Ref. 13); symbols: experiment.

dinal evolution of both the kinetic turbulent energy and the Reynolds shear stress obtained in the present LES to experimental data and the results of Druault et al. ${ }^{12}$ and Coiffet et $a .^{29}$ from their LESs of a turbulent mixing layer. These two simulations were performed in configurations very similar to the one retained in the present paper, namely, the inlet of the computational domain is located in a region where the flow is fully developed, e.g., far downstream of the trailing edge of the splitter plate. The main differences between these studies and the present work are the employed methods for generating the inlet conditions and the fact that the inlet sections of these two LES are in a region where the mixing layer has reached its self-similar state, contrary to the present paper in which the inlet is located in a region where only the mean flow is self-similar (see experimental values in Fig. 14). As described in the Introduction of the present paper, Druault $e t a l .{ }^{12}$ used data generated from hot-wire measurements obtained at several locations in the inlet plane. The results from Coiffet $e t a .^{29}$ are those obtained with random noise as inlet velocity fluctuations, preserving only the Reynolds stresses in the inlet domain. These authors tested this rather simple method, which is commonly used to perform simulations using a computational domain beginning at the (a)

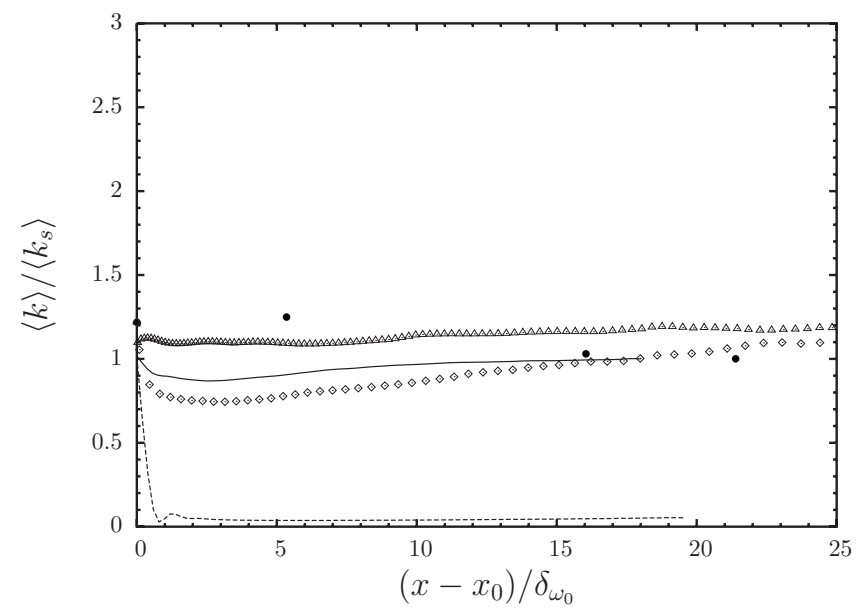

(b)

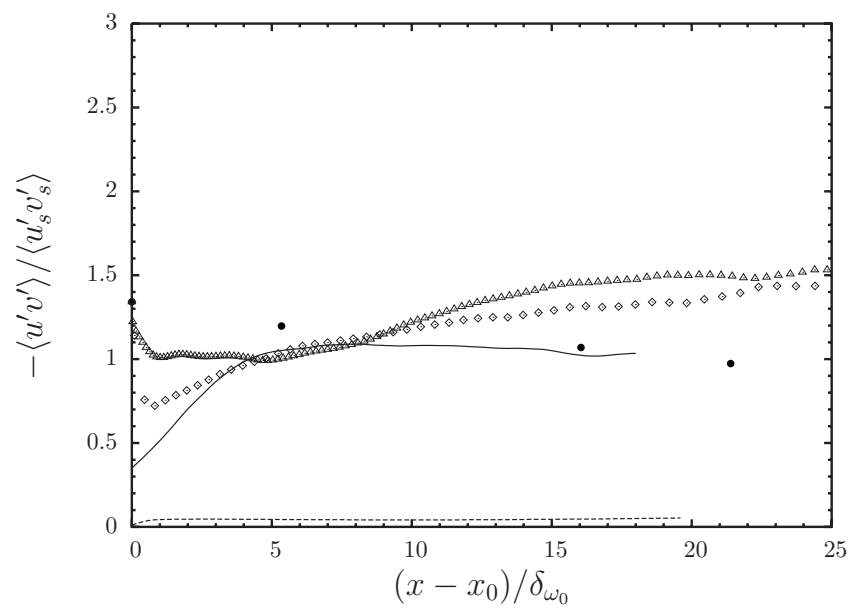

FIG. 14. Longitudinal evolution of (top) the turbulent kinetic energy $\langle k\rangle$ and (bottom) the shear stress $-\left\langle u^{\prime} v^{\prime}\right\rangle$ normalized by the experimental values obtained in the similitude region (furthest location) in the center of the shear layer $(y=0)$; open triangles: present computation; open diamonds: LES of Perret et al. (Ref. 13); solid line: LES of Druault et al. (Ref. 12) using hot-wire data as inflow condition; dashed line: LES of Coiffet et al. (Ref. 29) using random noise as inflow condition; black circles: experiment.

origin of the flow ${ }^{34-36}$ to evaluate its performance when the inlet domain is located in the self-similar region of the mixing layer. Results of Perret et al., ${ }^{13}$ in which no dynamical system is employed but which reproduce the full two-point correlation tensor, are also presented in Fig. 14. It should be noted here that the first and second moments are exactly the same for the present results, those from Perret et al. ${ }^{13}$ and the experimental data. It can be seen that the method employing random noise as inlet condition leads to a dramatic breakdown of the energy and Reynolds shear stress. This simulation thus completely fails to reproduce the targeted flow. The method proposed by Perret et al. ${ }^{13}$ which enables the reproduction of the first moments and the full two-point correlation tensor but still relies on random signal without the correct temporal phase, performs better but still exhibits a region just past the inlet where the energy and Reynolds shear stress levels drop and then increase slowly to reach the targeted levels. The method of Druault et al. ${ }^{12}$ based on the use of time history from hot-wire measurements still shows a 
slight decrease of the energy level. As this method is not designed to reproduce the correct shear stress, a region of adaptation in which this quantity evolves to reach the correct level still exists in the first quarter of the computational domain. After this region, the self-similar state is correctly reproduced. The present method enables the reproduction of the correct level at the entry and does not show any important decrease of the quantity levels. Compared to the results of Perret et al., ${ }^{13}$ it is demonstrated that reproducing the correct behavior of the large scale structures of the flow via the use of a LODS improves the quality of the simulation. However, it can be seen that the kinetic energy remains almost constant in the entire domain and that the level of the Reynolds shear stress increases at the end of the domain. The present simulation thus fails to reproduce the slow longitudinal decrease of the energy and Reynolds shear stress levels representing the fact that the flow reaches its self-similar state. However, it is still not clear whether the trend exhibited by the Reynolds shear stress is a consequence of the employed method for generating the inlet conditions or a sign that the mesh resolution is not optimal. This trend being retrieved both in the LES of Perret et al. ${ }^{13}$ and in the present computation, it may indicate that this is likely due to a problem of confinement of the flow in the downstream part of the computational domain

The spectral content of the simulated mixing layer is studied by comparing velocity spectra obtained at the center of the mixing layer with those measured by hot wires at two different streamwise locations (Figs. 15 and 16). Despite the lower statistical convergence of the numerical results and the expected LES filtering, spectra of the three velocity components show good agreement with the experimental results. Energy repartition in a part of the inertial range as well as in the low-frequency range is well reproduced, with the presence of dominant frequencies corresponding to those characteristic of the large scale Kelvin-Helmholtz structures. The rapid decrease of the energy and the departure from the experimental results around $f \delta_{\omega} / U_{m}=3$ correspond to the LES filtering of the small scale. Besides, these results show that the strong frequency peaks mentioned in the previous section have little influence on the downstream development of the simulated flow even in the upstream region (Fig. 15).

To investigate the spatial organization of the flow simulated by LES, two-point spatial correlations $R_{i i}\left(y, y^{\prime}\right)$ are computed and compared to experimental results. ${ }^{16}$ Figures 17 and 18 show the correlation tensors obtained at two streamwise locations for the three velocity components. Again, in spite of the lower statistical convergence (only the last 15000 time steps are retained for the statistics computation in the case of the LES, being equivalent to the time required for the flow to travel 6 times the length of the computational domain), agreement is obtained for correlation levels as well as correlation shapes. Thus, even in the upstream region of the computational domain, characteristics of the spatial organization of mixing layer flows, such as correlation length and negative levels of correlation, are reproduced by the simulation. These results show that no strong (a)

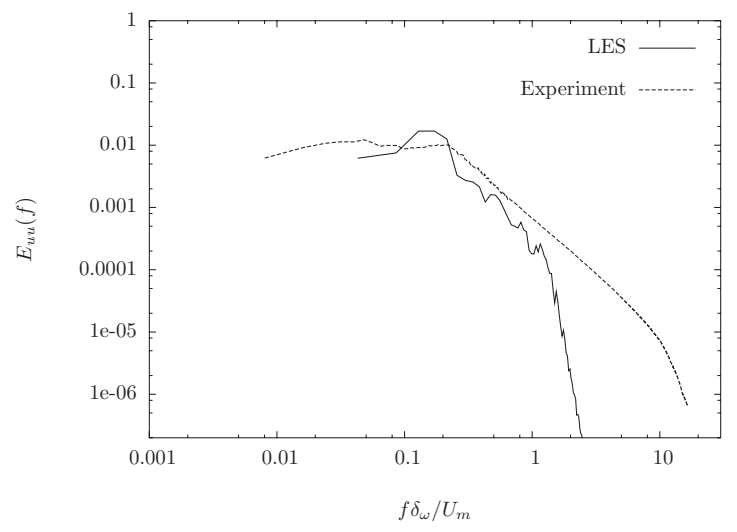

(b)

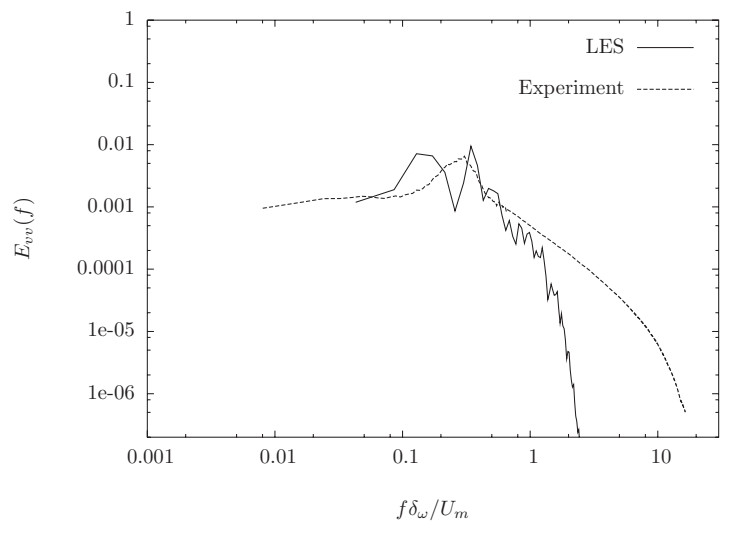

(c)

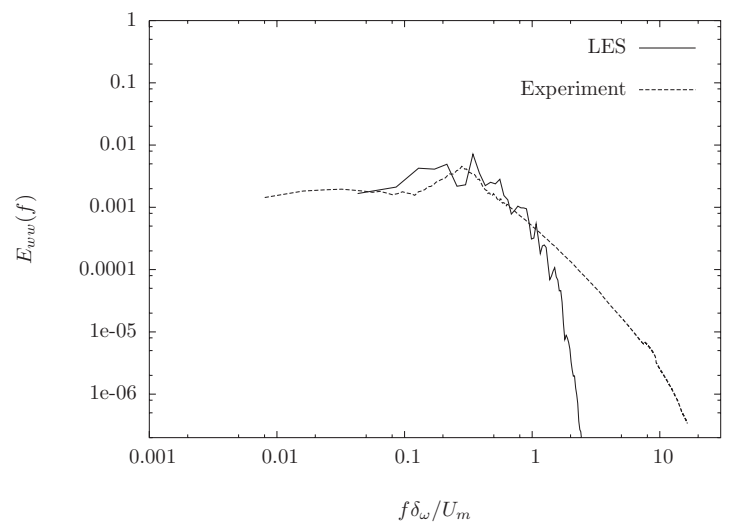

FIG. 15. Energy spectrum of (a) the longitudinal, (b) the vertical, and (c) the spanwise velocity components on the shear-layer axis at $\left(x-x_{0}\right) / \delta_{\omega 0}=5.3$ (solid line) of the LES results and (dashed line) from hot-wire measurements.

adjustment of the inlet conditions is needed for the computation to run and produce a flow with proper large-scale organization.

\section{CONCLUSION}

In this paper, a new method for generating turbulent inflow boundary conditions for LES has been presented. On the basis of previous studies conducted either by the authors or by other research groups, the present effort aims at proposing an innovative method to synthesize turbulent velocity 
(a)

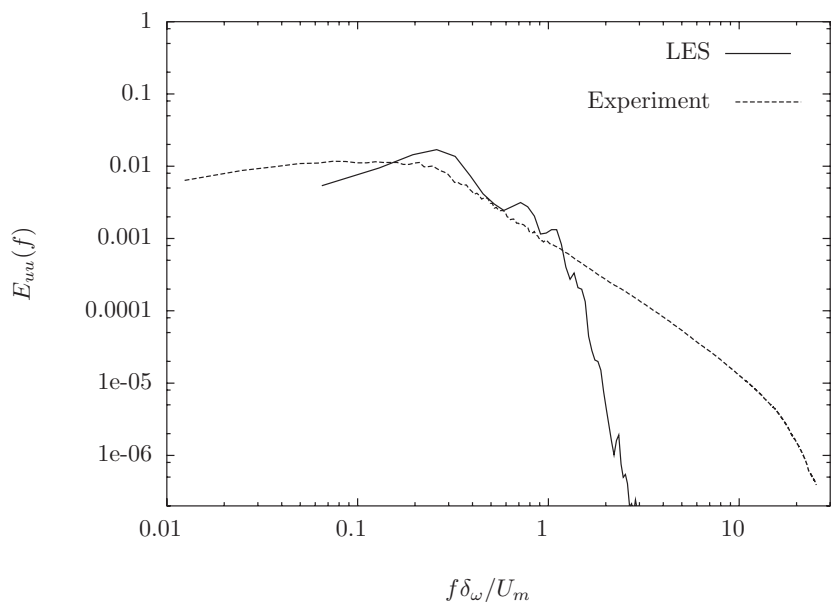

(b)

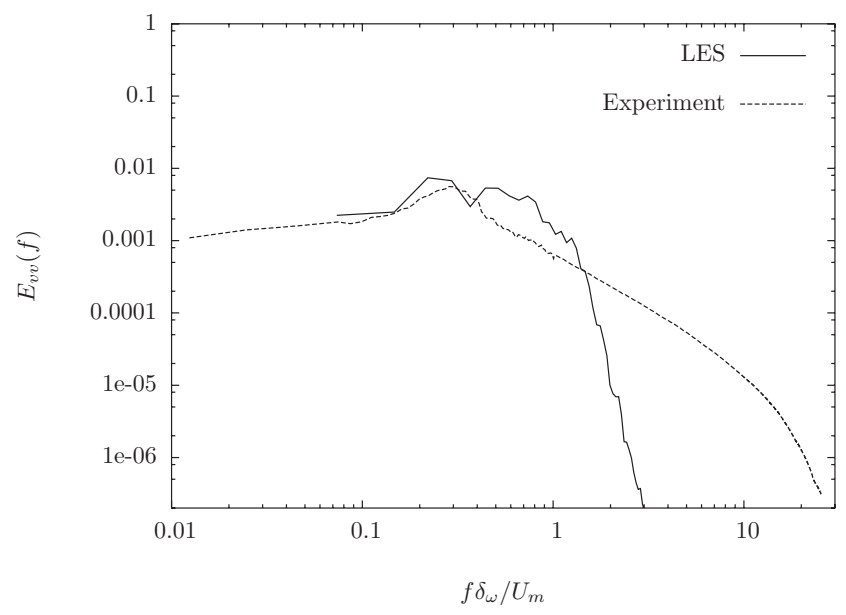

(c)

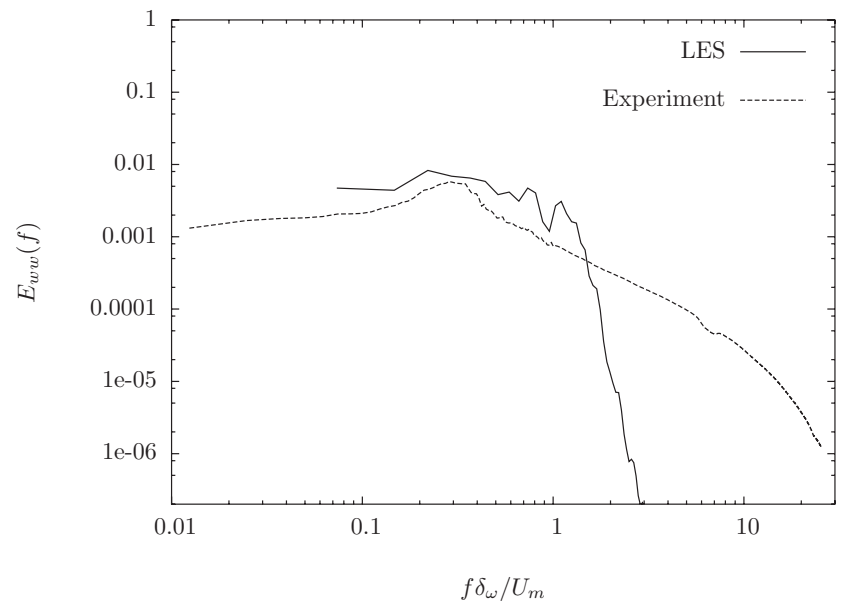

FIG. 16. Energy spectrum of (a) the longitudinal, (b) the vertical, and (c) the spanwise velocity components on the shear-layer axis at $\left(x-x_{0}\right) / \delta_{\omega 0}=21.4$ (solid line) of the LES results and (dashed line) from hot-wire measurements.

fields that match at best the targeted flow. For this purpose, generated velocity fields present the correct one- and twopoint spatial statistics and the correct spectral content. In addition, attention has been paid to the correct reproduction (a) $R_{u u}\left(y, y^{\prime}\right)$

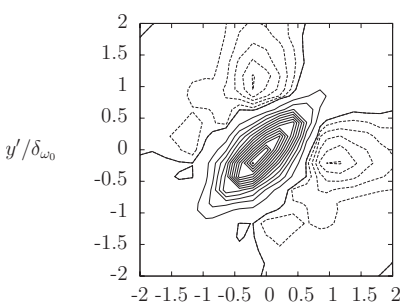

$y / \delta_{\omega_{0}}$

(c) $R_{v v}\left(y, y^{\prime}\right)$

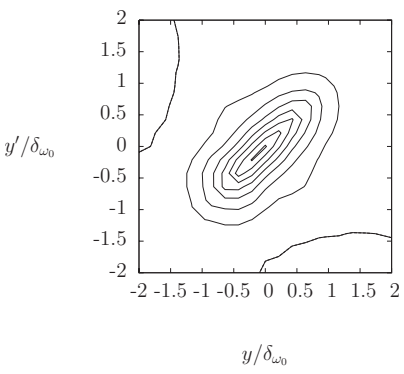

(e) $R_{w w}\left(y, y^{\prime}\right)$

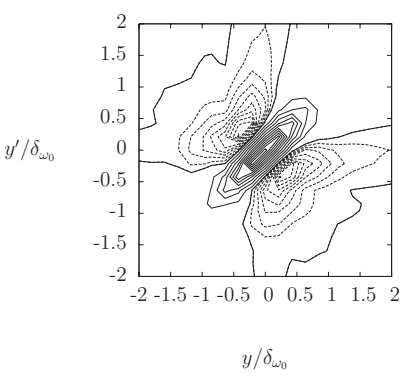

(b) $R_{u u}\left(y, y^{\prime}\right)$

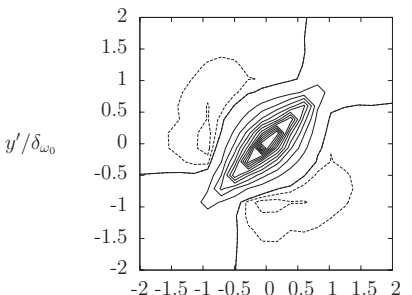

$y / \delta_{\omega_{0}}$

(d) $R_{v v}\left(y, y^{\prime}\right)$

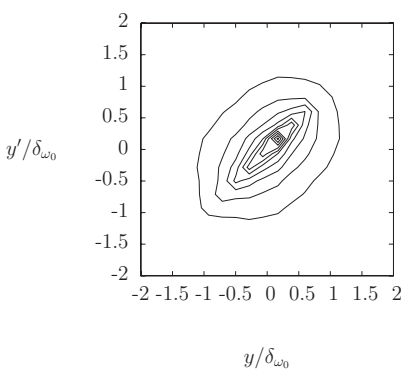

(f) $R_{w w}\left(y, y^{\prime}\right)$

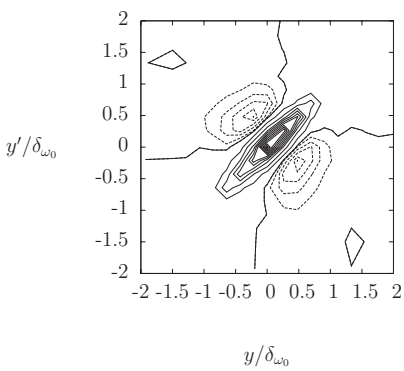

FIG. 17. Two-point correlation of the three velocity components obtained at $\left(x-x_{0}\right) / \delta_{\omega 0}=5.3$; left: LES; right: experiment; (solid) positive contours in increment of 0.5 and (dashed) negative contours in increment of 0.1 .

of the dynamics of the flow, this point being of crucial importance when dealing with the simulation of complex turbulent flows. To achieve this goal, an interface between an experimental database, which does not contain any time history of velocity signals, and the computational code is developed by using the POD combined to an original method designed to derive a LODS from an experimental database. ${ }^{14}$ This approach ensures the adaptability of the method: the proposed techniques indeed do not take advantage of any of the characteristics of the simulated flow and are thus not limited to the present test case. For this purpose, DT-SPIV has been chosen to acquire the database. Despite its experimental difficulties (e.g., set up and align four laser sheets and four cameras) and limitations (the same as the classical PIV technique plus the issue of choosing the proper set of parameters to be able to correctly compute the time derivative), this measurement technique has proven to be a reliable tool to investigate a wide variety of turbulent flows, giving access not only to three-component velocity fields but also to their temporal derivatives. The present method of inflow condition generation can thus be applied once one is able to perform a DT-SPIV experiment in the configuration of interest. This 
(a) $R_{u u}\left(y, y^{\prime}\right)$

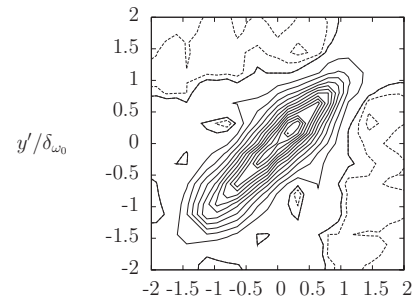

$y / \delta_{\omega_{0}}$

(c) $R_{v v}\left(y, y^{\prime}\right)$

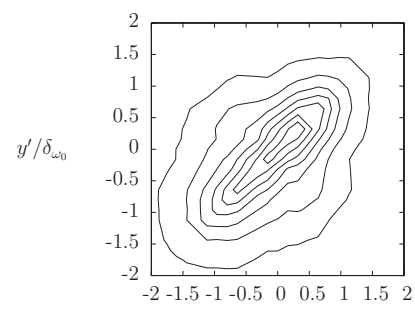

$y / \delta_{\omega_{0}}$

(e) $R_{w w}\left(y, y^{\prime}\right)$

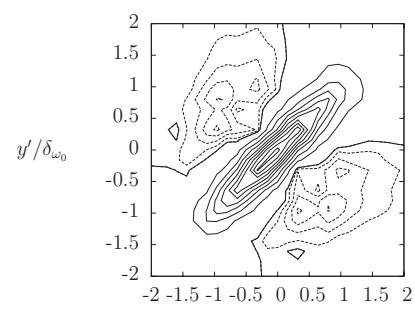

$y / \delta_{\omega_{0}}$ (b) $R_{u u}\left(y, y^{\prime}\right)$

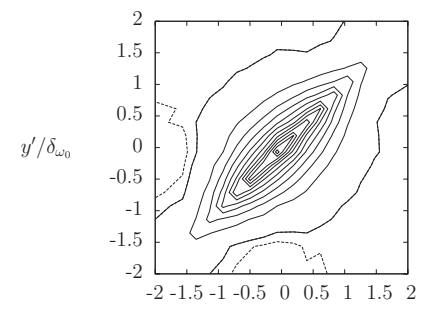

$y / \delta_{\omega_{0}}$

(d) $R_{v v}\left(y, y^{\prime}\right)$

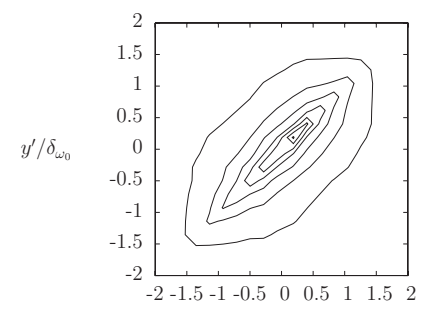

$y / \delta_{\omega_{0}}$

(f) $R_{w w}\left(y, y^{\prime}\right)$

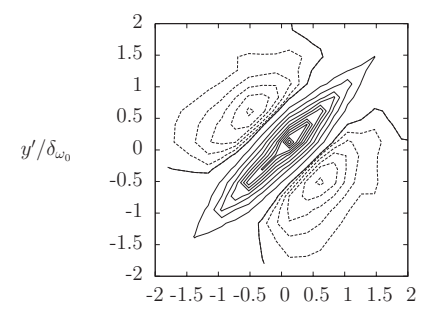

$y / \delta_{\omega_{0}}$

FIG. 18. Two-point correlation of the three velocity components obtained at $\left(x-x_{0}\right) / \delta_{\omega 0}=21.4$; left: LES; right: experiment; (solid) positive contours in increment of 0.5 and (dashed) negative contours in increment of 0.1 .

can be of particular interest when inflow conditions are required to perform computation of highly complex flows such as multiple swirling jets, for instance. 9,37

In the proposed approach, attention has been paid not only to the correct modeling of the spatiotemporal dynamics (via a LODS and randomly generated time-varying signals) but also to the proper spatial adjustment of the experimental data to the numerical grid. In particular, a combination of different techniques based on POD and signal processing has been employed to deal with the specific boundary conditions retained in the final LES, namely, free-slip conditions on the horizontal boundaries and periodic conditions in the homogeneous spanwise direction. In order to validate this new methodology, LES of a plane turbulent mixing layer has been performed and carefully investigated. Detailed comparisons with experimental data, including spectral analysis and oneand two-point statistics computation, have been provided.

This numerical test demonstrates the feasibility and the reliability of the proposed method. It also opens new routes of investigation by highlighting some remaining issues. As far as the database generation is concerned, the present study showed that the number of modes that can be retained to

derive a LODS is limited by the presence of noise in the DT-SPIV measurements. The use of new experimental techniques, such as TR-PIV that now becomes available to perform accurate measurements in turbulent flows, can improve the time-derivative computation. The LODS derivation method can be improved too in order to increase the model stability. Modeling of the temporal dynamics of the high order modes also requires further developments to model their influence and interaction with modes that are taken into account in the LODS.

In addition, more complex flow configurations, such as wall-bounded and compressible flows, have to be considered to fully validate the proposed method and require further developments. For example, in wall-bounded flows, one can consider boundary conditions normal both to the mean flow (e.g., inlet boundary condition) but also wall-parallel boundary conditions used to avoid the expensive computation of the near wall region. In these planes, modeling requirements may be different as the spatial scales in the wall-normal direction and the one in the streamwise direction are different, and several different spatial scales are involved in each plane. Moreover, if two boundary conditions are modeled, their phase coupling must be correctly handled. Compressible flows raise the problem of the choice of the proper variables retained to perform the inner product in the POD approach as well as the issue of acoustic coupling between the flow and its inlet and outlet sections.

Finally, this first attempt at combining an experimental database to a LES code via a LODS suggests that low-order models could be considered as a way to provide unsteady conditions at a RANS/LES interface in hybrid approaches. If one were able to generate the full two-point correlation tensor (e.g., three components, three directions) from the statistical information provided by the RANS computation, LODS derivation via a classical POD-Galerkin approach would be feasible. This dynamical model could then serve as an inflow condition generator for the LES part.

\section{ACKNOWLEDGMENTS}

The authors acknowledge the financial support provided by the French Ministry of Defense. The authors also wish to thank P. Braud, C. Fourment, and Dr. L. David for their contribution to the experimental part of this work.

\footnotetext{
${ }^{1}$ P. Sagaut, S. Deck, and M. Terracol, Multiscale and Multiresolution Approaches in Turbulence (Imperial College Press, London, 2006).

${ }^{2}$ S. Lee, S. Lele, and P. Moin, "Simulation of spatially evolving compressible turbulence and application of Taylor's hypothesis," Phys. Fluids A 4, 1521 (1992).

${ }^{3}$ H. Le, P. Moin, and J. Kim, "Direct numerical simulation of turbulent flow over a backward facing step," J. Fluid Mech. 330, 349 (1997).

${ }^{4}$ N. D. Sandham, Y. F. Yao, and A. Lawal, "Large-eddy simulation of transonic turbulent flow over a bump," Int. J. Heat Fluid Flow 24, 584 (2003).

${ }^{5}$ N. Jarrin, S. Benhamadouche, D. Laurence, and R. Prosser, "A syntheticeddy-method for generating inflow conditions for large-eddy simulations," Int. J. Heat Fluid Flow 27, 585 (2006).

${ }^{6}$ T. S. Lund, X. Wu, and K. D. Squires, "Generation of inflow data for spatially-developing boundary layer simulations," J. Comput. Phys. 140, 233 (1998).

${ }^{7}$ A. Ferrante and S. E. Elghobashi, "A robust method for generating inflow conditions for direct simulations of spatially-developing turbulent bound-
} 
ary layers," J. Comput. Phys. 198, 372 (2004).

${ }^{8}$ N. Li, E. Balaras, and U. Piomelli, "Inflow conditions for large-eddy simulations of mixing layers," Phys. Fluids 12, 935 (2000).

${ }^{9}$ J. U. Schlüter, H. Pitsch, and P. Moin, "LES inflow conditions for coupling with Reynolds averaged flow solvers," AIAA J. 42, 478 (2004).

${ }^{10}$ A. Keating, U. Piomelli, and E. Balaras, "A priori and a posteriori tests of inflow conditions for large-eddy simulation," Phys. Fluids 16, 4696 (2004).

${ }^{11}$ P. S. Johansson and H. I. Andersson, "Generation of inflow data for inhomogeneous turbulence," Theor. Comput. Fluid Dyn. 18, 371 (2004).

${ }^{12}$ P. Druault, S. Lardeau, J. P. Bonnet, F. Coiffet, J. Delville, E. Lamballais, J. F. Largeau, and L. Perret, "Generation of three-dimensional turbulent inlet conditions for large-eddy simulation," AIAA J. 42, 447 (2004).

${ }^{13}$ L. Perret, R. Manceau, J. Delville, and J. P. Bonnet, "Generation of turbulent inflow conditions for large eddy simulation from stereoscopic PIV measurements," Int. J. Heat Fluid Flow 27, 576 (2006).

${ }^{14}$ L. Perret, E. Collin, and J. Delville, "Polynomial identification of POD based low-order dynamical system," J. Turbul. 7, 1 (2006).

${ }^{15}$ L. Perret, P. Braud, C. Fourment, L. David, and J. Delville, "3-component acceleration field measurement by dual-time stereoscopic particle image velocimetry," Exp. Fluids 40, 813 (2006).

${ }^{16}$ L. Perret, "Etude du couplage instationnaire calculs-expérience en écoulements turbulents," Ph.D. thesis, Université de Poitiers, 2004.

${ }^{17}$ S. M. Soloff, R. J. Adrian, and Z. C. Liu, "Distorsion compensation for generalized stereoscopic particle image velocimetry," Meas. Sci. Technol. 8, 1441 (1997)

${ }^{18}$ L. P. Bernal and A. Roshko, "Streamwise vortex structures in plane mixing layers," J. Fluid Mech. 170, 499 (1986).

${ }^{19}$ J. Delville, L. Ukeiley, L. Cordier, J. P. Bonnet, and M. Glauser, "Examination of large-scale structures in a turbulent plane mixing layer. Part 1. Proper orthogonal decomposition," J. Fluid Mech. 391, 99 (1999).

${ }^{20}$ J. L. Lumley, in Atmospheric Turbulence and Radio Wave Propagation, edited by A. M. Yaglom and V. L. Tatarsky (Nauka, Moscow, 1967), pp. $166-178$

${ }^{21}$ G. Berkooz, P. Holmes, and J. L. Lumley, "The proper orthogonal decomposition in the analysis of turbulent flows," Annu. Rev. Fluid Mech. 25, 539 (1993).

${ }^{22}$ L. Sirovich, "Turbulence and the dynamics of coherent structures, Part 1: Coherent structures," Q. Appl. Math. 45, 561 (1987).

${ }^{23}$ W. C. Reynolds and A. K. M. F. Hussain, "The mechanics of an organized wave in turbulent shear flow. Part 3. Theoretical models and comparisons with experiments," J. Fluid Mech. 54, 263 (1972).
${ }^{24}$ N. Aubry, P. Holmes, J. Lumley, and E. Stone, "The dynamics of coherent structures in the wall region of a turbulent boundary layer," J. Fluid Mech. 192, 115 (1988).

${ }^{25}$ L. Ukeiley, L. Cordier, R. Manceau, J. Delville, M. Glauser, and J. P. Bonnet, "Examination of large-scale structures in a turbulent plane mixing layer. Part 2. Dynamical systems model," J. Fluid Mech. 441, 67 (2001).

${ }^{26}$ C. Braud, D. Heitz, G. Arroyo, L. Perret, and J. Delville, "Lowdimensional analysis, using POD, for two mixing layer? Wake interactions," Int. J. Heat Fluid Flow 25, 351 (2004).

${ }^{27}$ J. P. Bonnet, J. Delville, M. N. Glauser, R. A. Antonia, D. K. Bisset, D. R. Cole, H. E. Fiedler, J. H. Garem, D. Hilberg, J. Jeong, N. K. R. Kevlahan, L. S. Ukeiley, and E. Vincendeau, "Collaborative testing of eddy structure identification methods in free turbulent shear flows," Exp. Fluids 25, 197 (1998).

${ }^{28}$ J. O. Hinze, Turbulence, 2nd ed. (McGraw-Hill, New York, 1975).

${ }^{29} \mathrm{~F}$. Coiffet, J. Delville, and E. Lamballais, in Advances in Turbulence IX: Proceedings of the Ninth European Turbulence Conference, edited by I. P. Castro, P. E. Hancock, and T. G. Thomas (CIMNE, Barcelona, 2002).

${ }^{30} \mathrm{U}$. Franke, International Conference on Acoustics, Speech Signal Processing (IEEE, New York, 1987).

${ }^{31}$ M. V. Droogenbroeck, "Traitement d'images numériques au moyen d'algorithmes utilisant la morphologie mathématique et la notion d'objet: Application au codage," Ph.D. thesis, Catholic University of Louvain, 1994.

${ }^{32}$ P. Druault and J. Delville, "Representation of the spatial correlation tensor of the velocity in free turbulent flows," C. R. Acad. Sci., Ser. IIb Mec. 328, 135 (2000).

${ }^{33}$ F. Archambeau, N. Mehitoua, and M. Sakiz, "Code_Saturne: a finite volume code for the computation of turbulent incompressible flows," Int. J. Finite Volumes 1, 1 (2004).

${ }^{34}$ P. Comte, J. H. Silvestrini, and P. Bégou, "Streamwise vortices in largeeddy simulations of mixing layers," Eur. J. Mech. B/Fluids 17, 615 (1998).

${ }^{35}$ C. Tenaud, D. Pellerin, A. Dulieu, and L. T. Phuoc, "Large-eddy simulations of a spatially developing incompressible 3D mixing layers using the $v-\omega$ formulation," Comput. Fluids 34, 67 (2005).

${ }^{36}$ W. A. McMullan, S. Gao, and C. M. Coats, "A comparative study of inflow conditions for two- and three-dimensional spatially developing mixing layers using large-eddy simulation,” Int. J. Numer. Methods Fluids 55, 589 (2007).

${ }^{37}$ E. J. Gutmark, S. Verfaillie, J. P. Bonnet, and F. Grindstein, "Linear stochastic estimation of a swirling jet," AIAA J. 44, 457 (2006). 